\title{
Effect of Transcranial Direct Current Stmulation on the Response Inhibition in Patient with Obsessive Compulsive Disorder
}

\author{
Mahsa Shahmohammadi Kaleybar ${ }^{1 *}$, Hasan Bafandeh ${ }^{2}$, Rahim Yousefi' ${ }^{2}$, Reza Rahbaran ${ }^{3}$ \\ ${ }^{1}$ Department of Cognitive Psychology, Azarbaijan Shahid Madani University, Tabriz, Iran \\ ${ }^{2}$ Department of Psychology, Azarbaijan Shahid Madani University, Tabriz, Iran \\ ${ }^{3}$ Department of Clinical Psychology, Tabriz Branch, Islamic Azad University, Tabriz, Iran
}

\section{A BSTRACT}

Introduction: Transcranial Direct Current Stimulation (tDCS) can improve or impair the function of the brain. Deficiency in response inhibition has been considered as one of the causes of psychiatric disorders, such as obsessive-compulsive disorder (OCD). The present investigation scrutinized the effect of transcranial direct current stimulation through simultaneous uni-side anodal on response inhibition in patients suffering from OCD. Materials and Methods: The participants were 25 patients who referred to Counseling Center of the Azarbaijan Shahid Madani University in 1396. The participants were divided in two groups and received two different types of stimulations; anodal and sham stimulations on F3 area as well as cathode stimulation on the orbitofrontal cortex (ten 20-minute 2mA tDCS sessions). Results: The results revealed that application of tDCS through simultaneous anodal significantly improved response inhibition in patients suffering from OCD. Conclusion: The findings have shown that application of anodal tDCS can improve the response inhibition in OCD patients, possibly via enhancement of excitability in the prefrontal cortex.

*Corresponding Author: Mahsa Shahmohammadi Kaleybar

E-mail: Mahsa Shahmohammadi773@yahoo.com 
اثربخشى تحريك الكتريكى مستقيم فراجمجمهاى بر بازدارى ياسخ در بيماران مبتلا به اختلال وسواس فكرى -عملى

$$
\begin{aligned}
& \text { مهسا شاه محمدى كليبر"'، حسن بافنده'، رحيم يوسفى '، رضا رهبران" } \\
& \text { احروه روانشناسى شناختى، دانشخاه شهيد مدنى آذربايجان، تبريز، ايران } \\
& \text { rَّروه روانشناسى، دانشعاه شهيد مدنى آذربايجان، تبريز، ايران }
\end{aligned}
$$

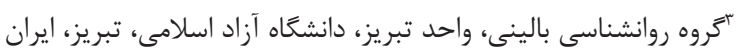

كليد وازمها:

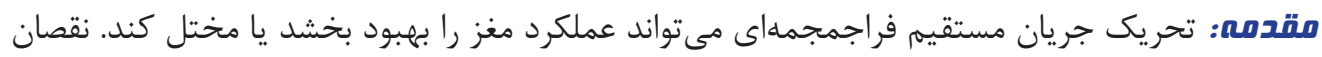

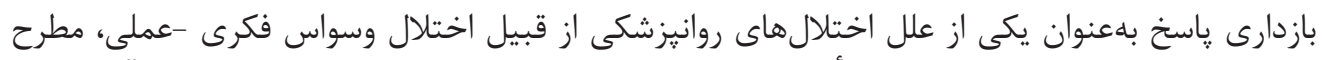

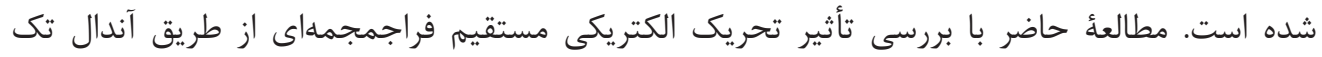

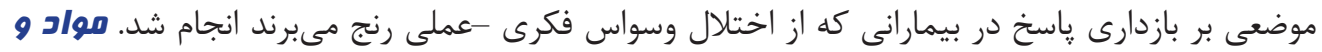

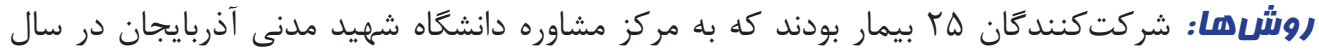

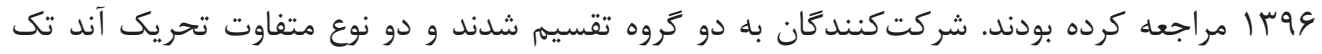

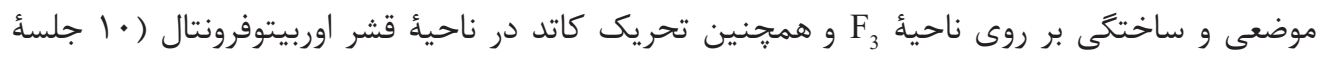

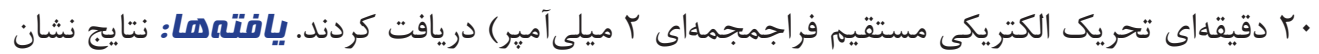

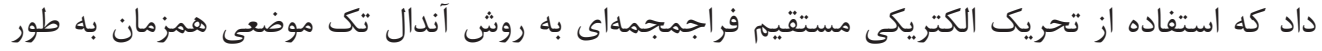

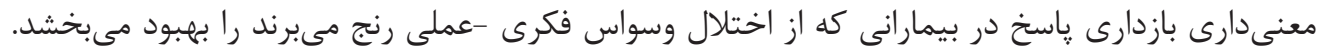

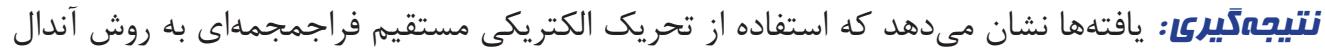

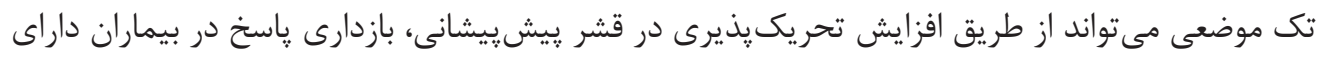

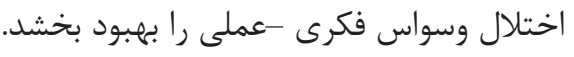

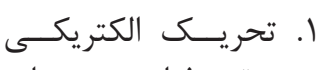

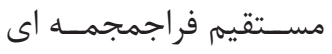

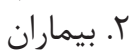

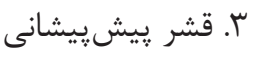

* نويسنده مسئول: مهسا شاه محمدى كليبر آدرس الكترونيكى: MahsaShahmohammadi773@yahoo.com 


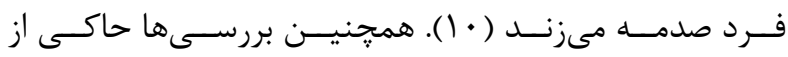

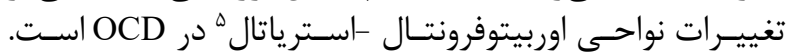

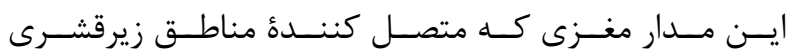

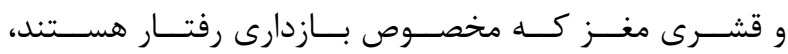

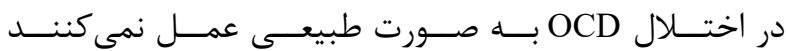
(V)

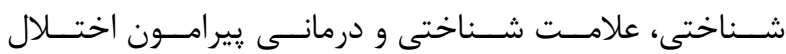

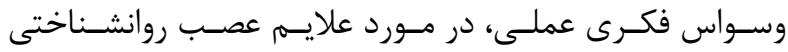

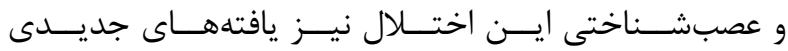

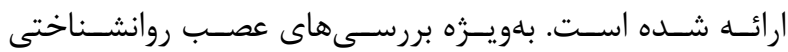

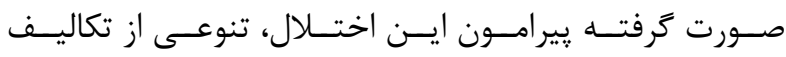

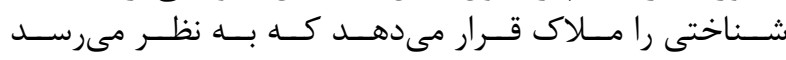

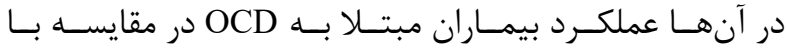

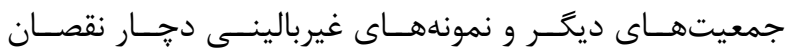

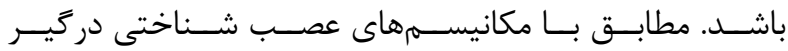

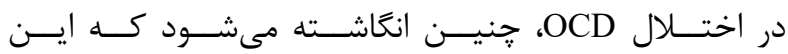

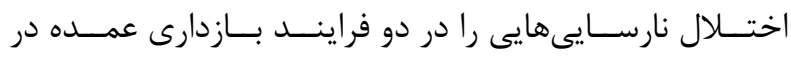

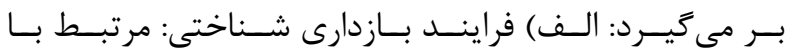

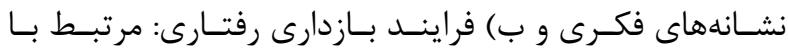

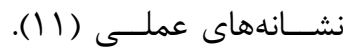

نارسـايىهاى موجـود در ايسن دو سيسـتم بـازدارى نــه تنهــا

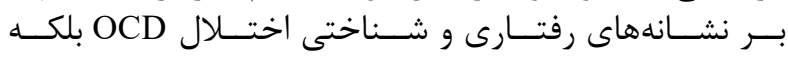

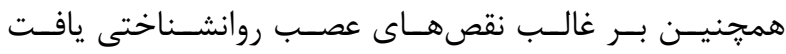

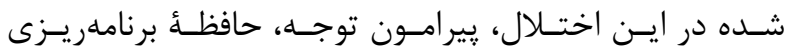

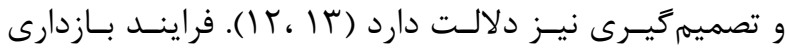

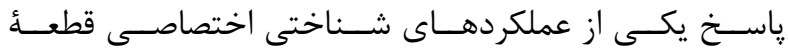

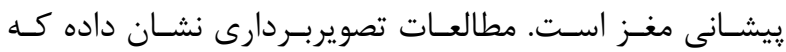

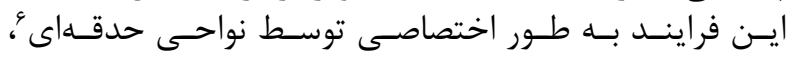

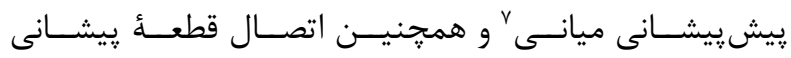

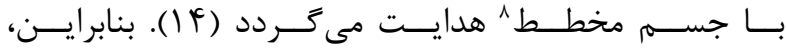

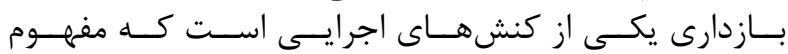

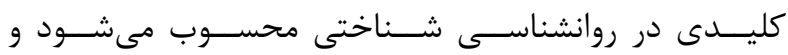

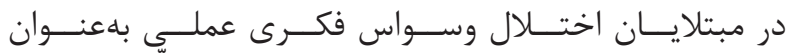

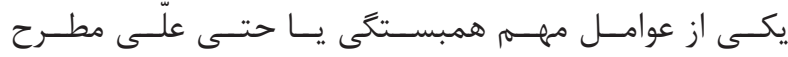

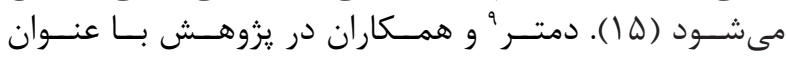

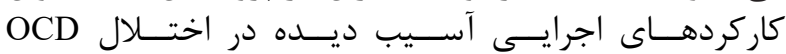

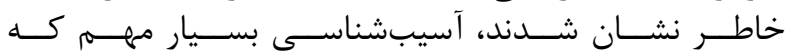

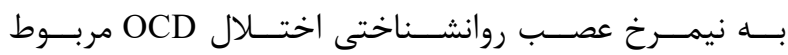

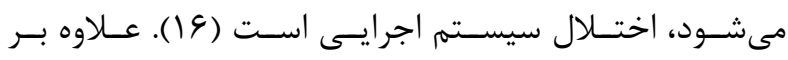

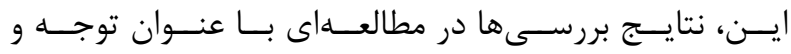

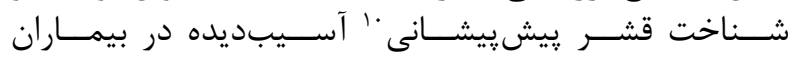

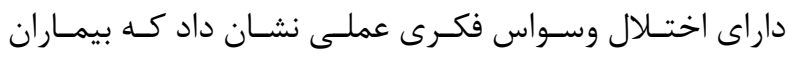

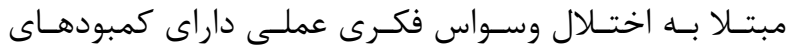

شـاختى " مىباشــند (IV)

${ }^{1}$ Obsessive- compulsive disorder

${ }^{2}$ Neurotransmitters

${ }^{3}$ Executive function

${ }^{4}$ Frontal-striatal

${ }^{5}$ Orbitofrontal-striatal

${ }^{6}$ Orbitofrontal

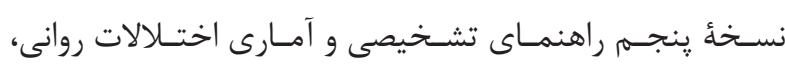

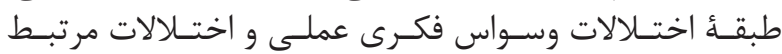

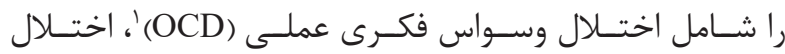

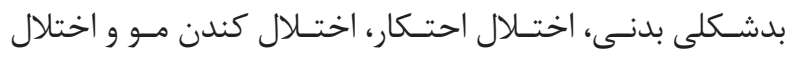

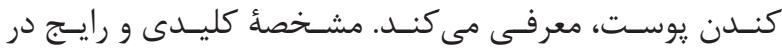

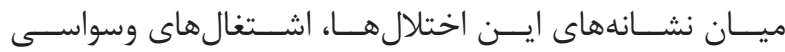

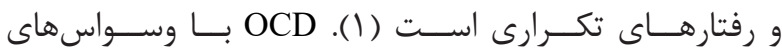

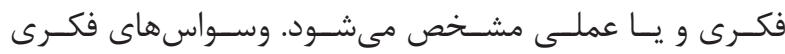

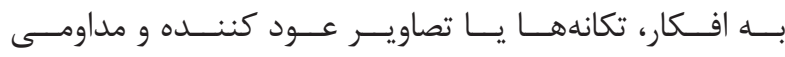

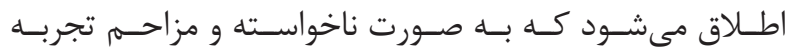

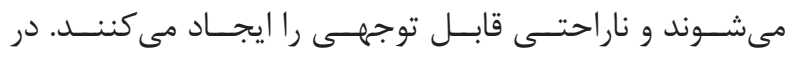

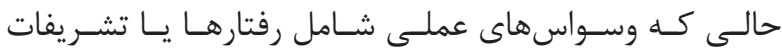

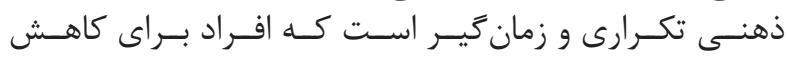

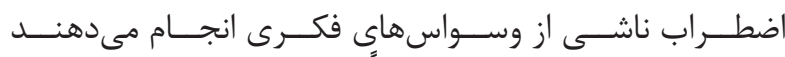

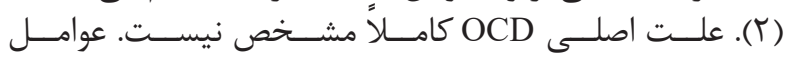

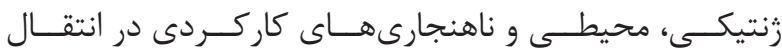

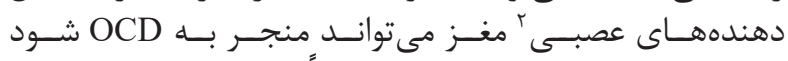

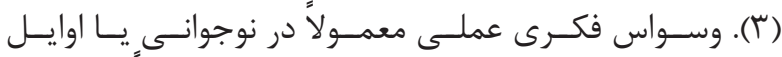

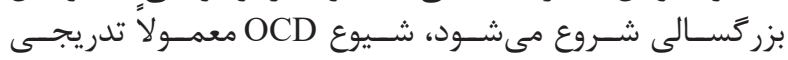

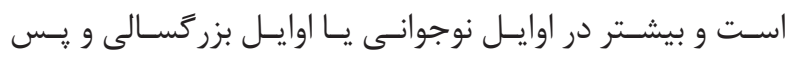

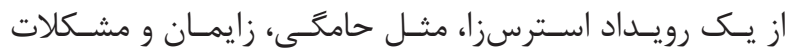

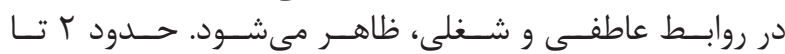

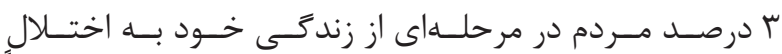

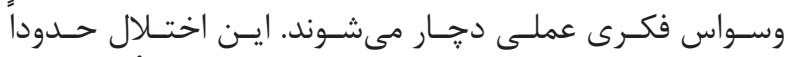

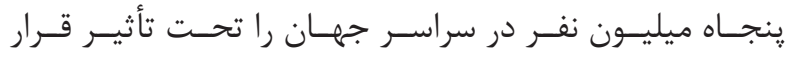

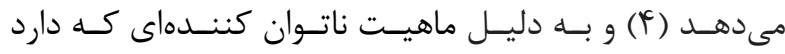

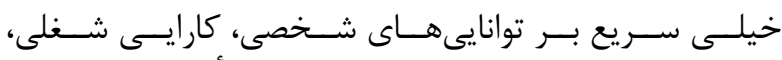

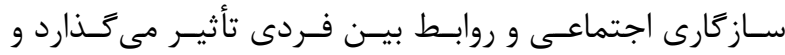

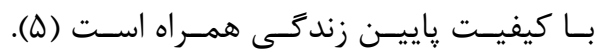

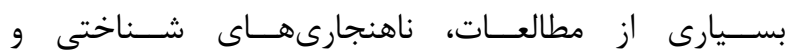

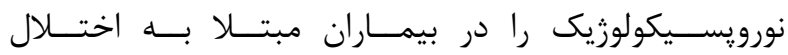

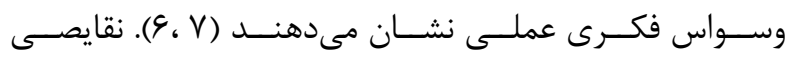

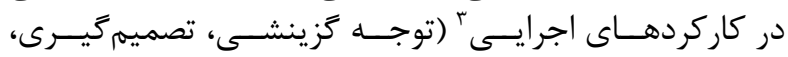

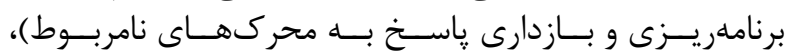

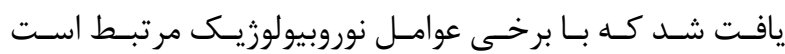

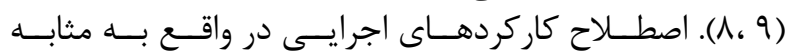

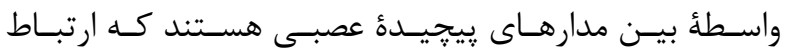

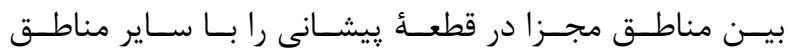

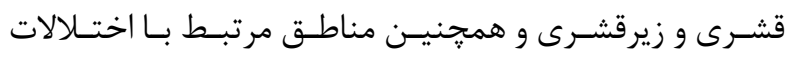

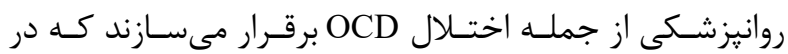

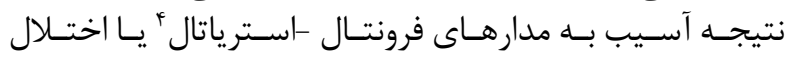

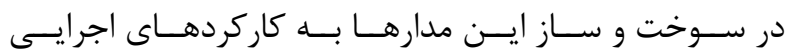

${ }^{7}$ Medial frontal

${ }^{8}$ Striatum

${ }^{9}$ Demter

${ }^{10}$ Profonetal cortex

${ }^{11}$ Cognitive deficits 


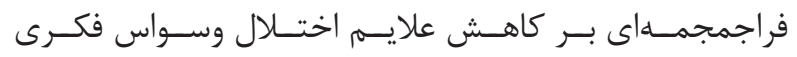

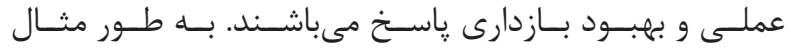

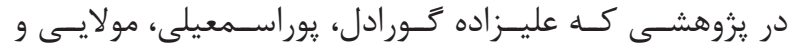

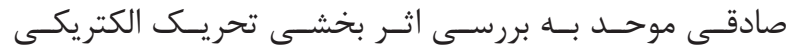

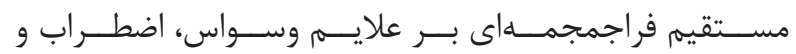

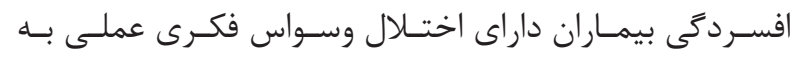

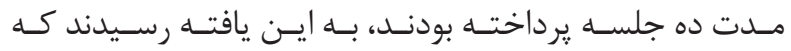

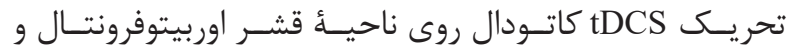

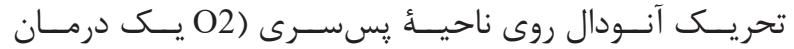

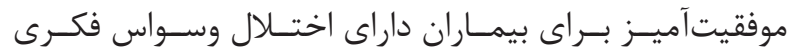

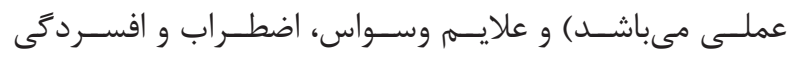

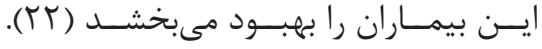

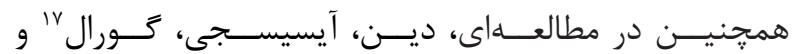

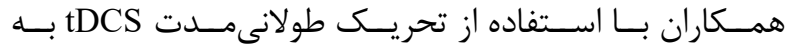

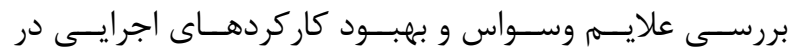

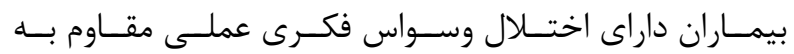

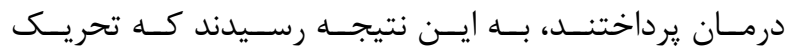

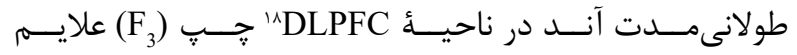

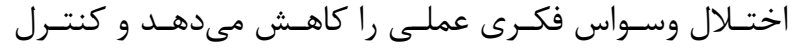

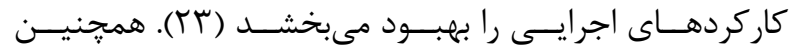

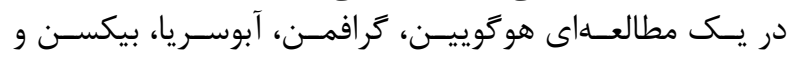

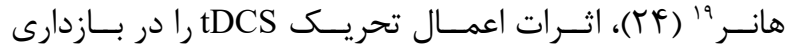

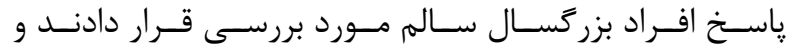

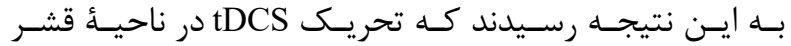

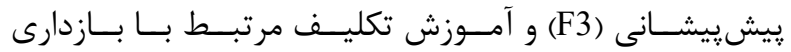

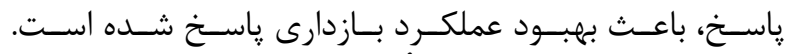

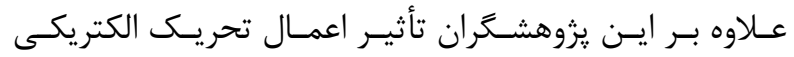

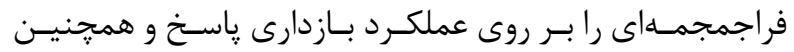

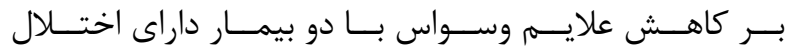

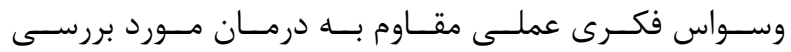

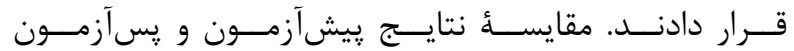

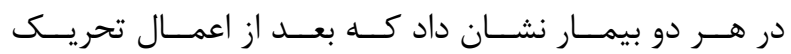

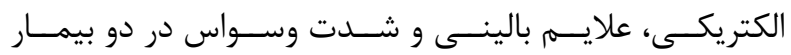

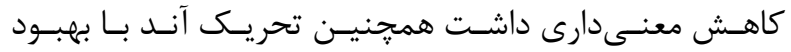

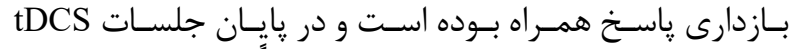

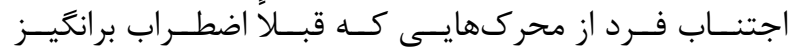

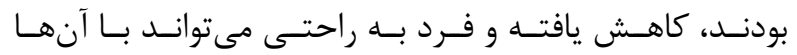

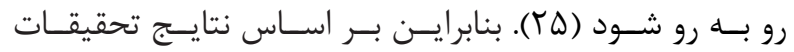

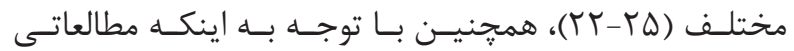

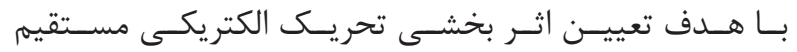

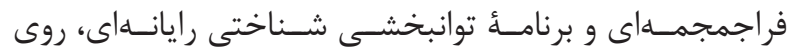

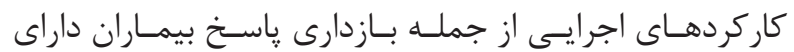

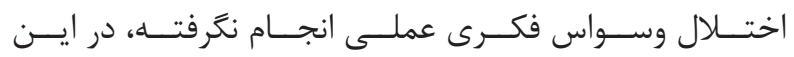

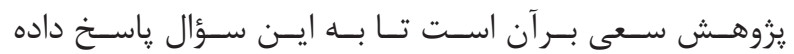

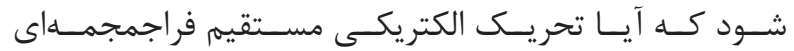

\footnotetext{
${ }^{12}$ Buhlmann

${ }^{13}$ Bannon

${ }^{14}$ Transcranial direct current stimulation

${ }^{15}$ Anode
}

در بررسىهاى ديكر كه هدفش آموزش تمركز شناختى روى بـاري

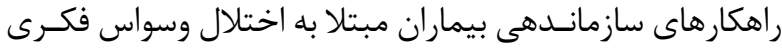

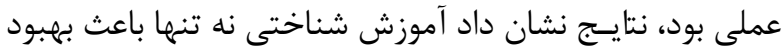

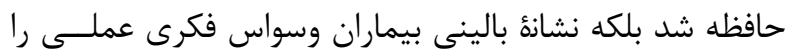

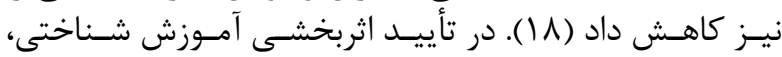

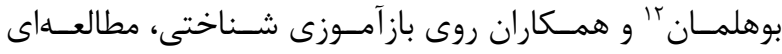

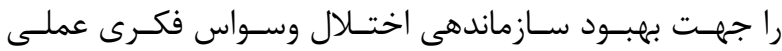

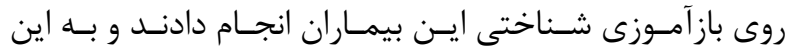

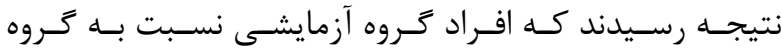

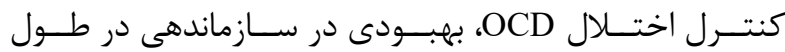

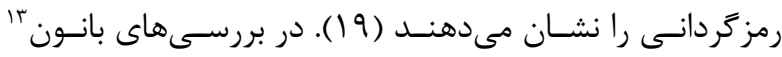

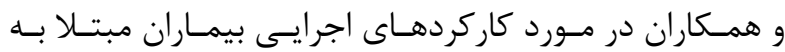

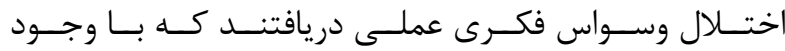

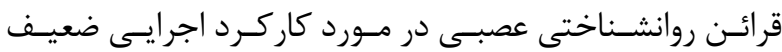

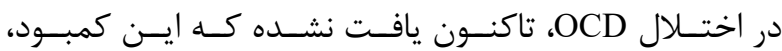

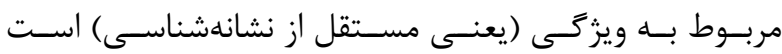

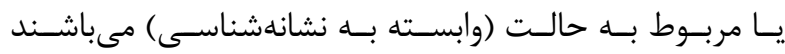

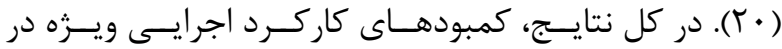

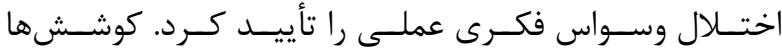

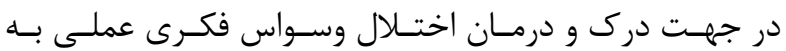

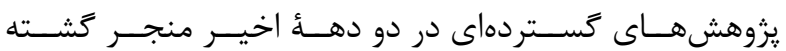

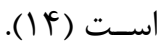

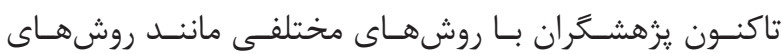

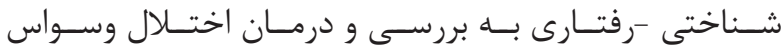

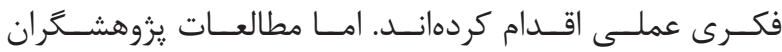

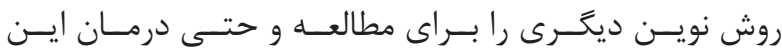

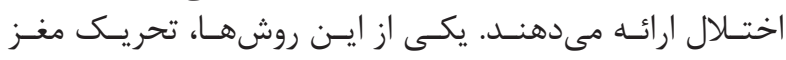

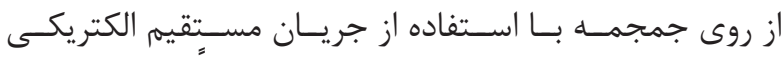
(tDCS)

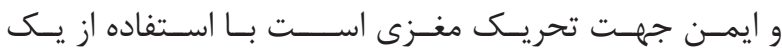

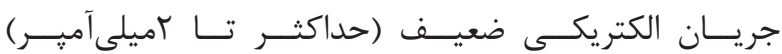

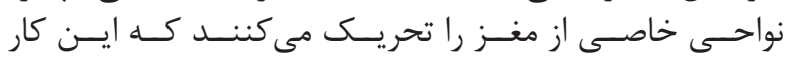

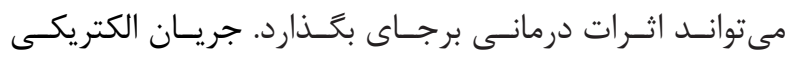

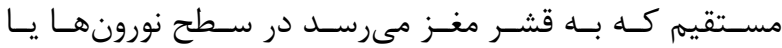

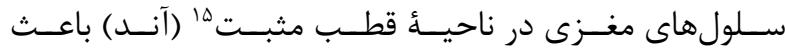

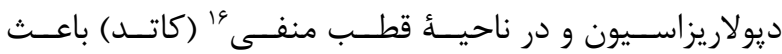

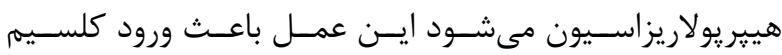

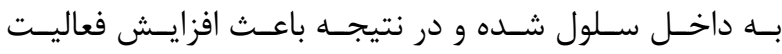

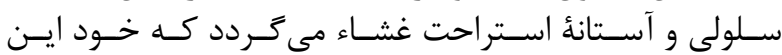

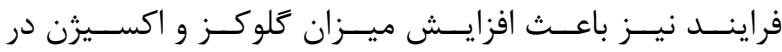

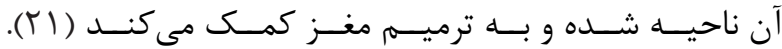

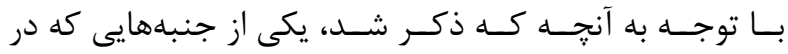

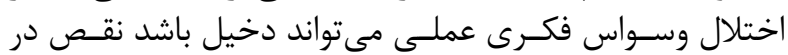

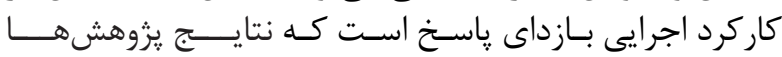

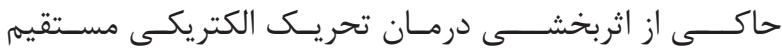

${ }^{16}$ Cathode

${ }^{17}$ Dinn, Aycicegi, Goral

${ }^{18}$ Dorsolateral prefrontal cortex

${ }^{19}$ Hogeveen, Grafman, Aboseria, Bikson, Hauner and David 


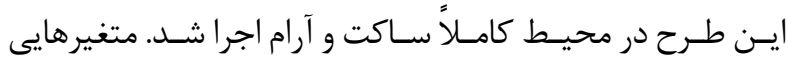

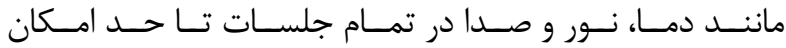

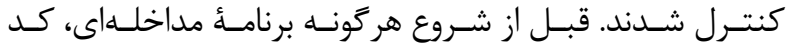

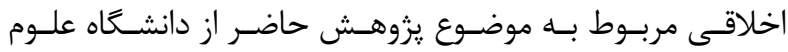

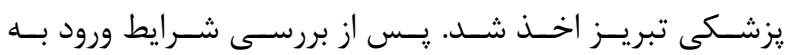

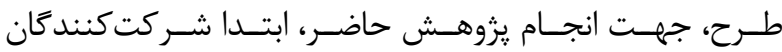

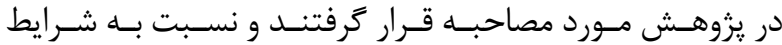

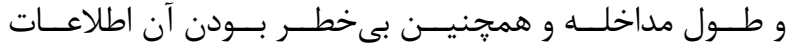

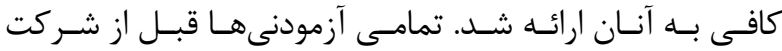

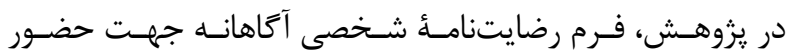

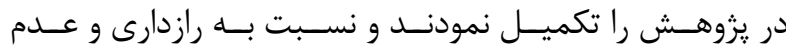

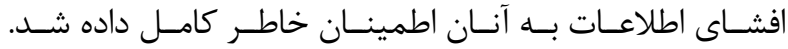

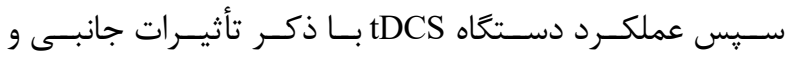

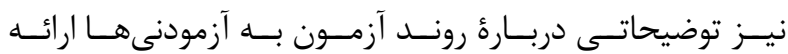

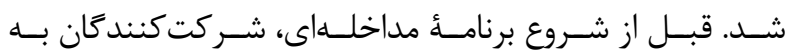

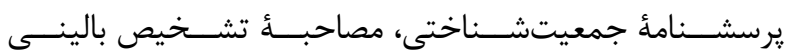

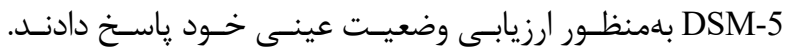

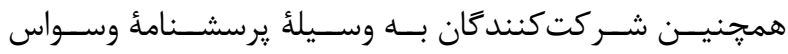

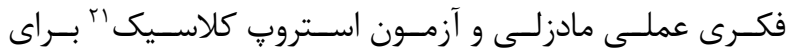

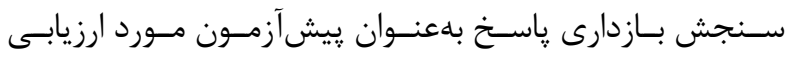

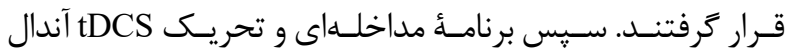

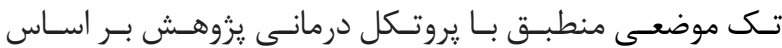

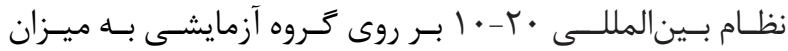

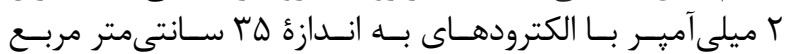

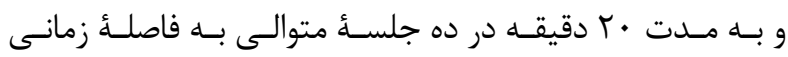

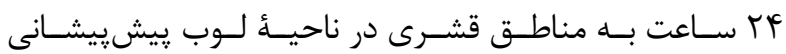

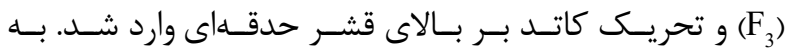

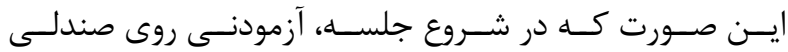

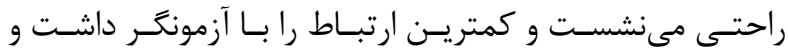

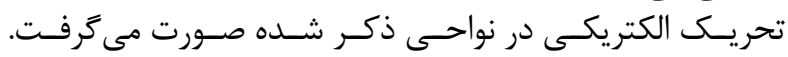

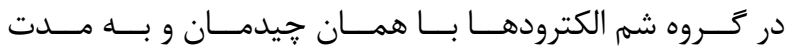

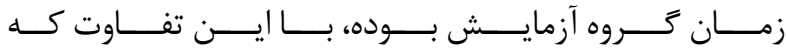

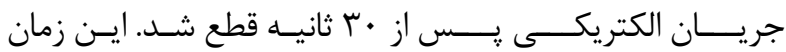

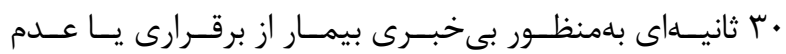

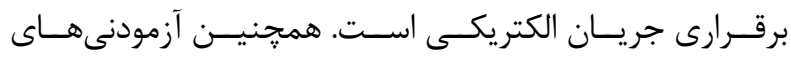

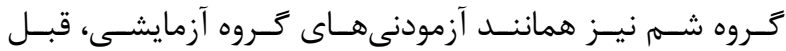

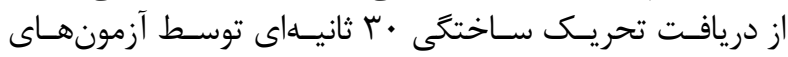

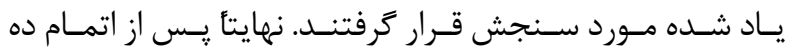

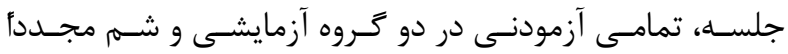

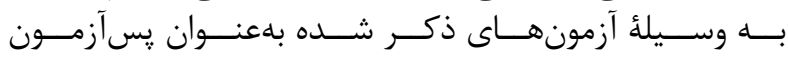

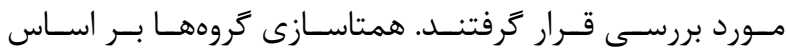

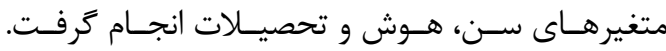

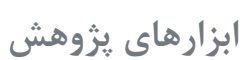

$$
\begin{aligned}
& \text { الف - آزمون استروب كلاسيك ترّى }
\end{aligned}
$$

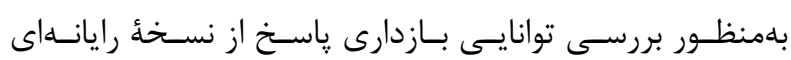

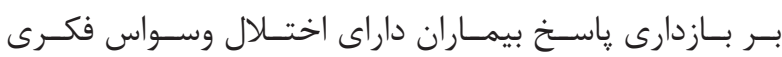

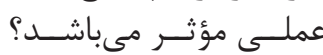

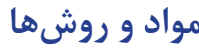

جامعلة آمارى و نمونه

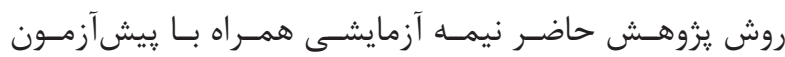

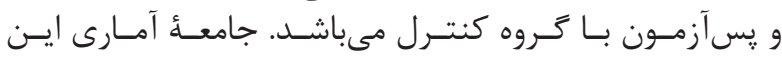

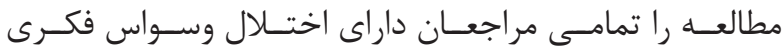

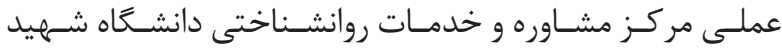

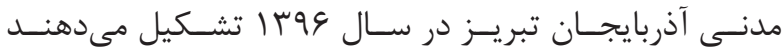

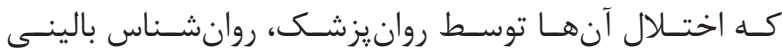

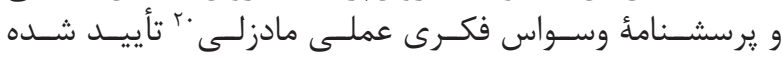

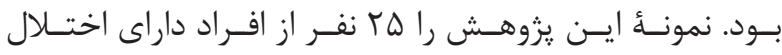

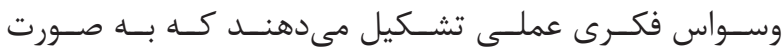

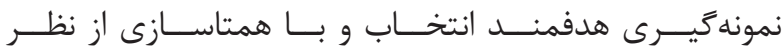

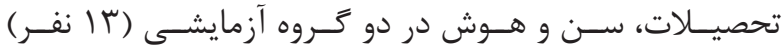

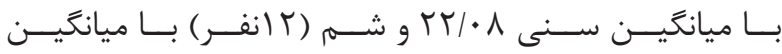

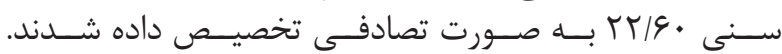

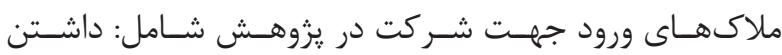

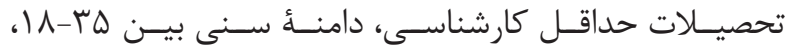

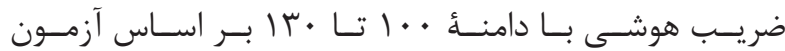

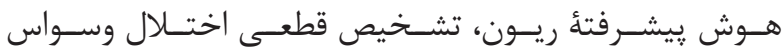

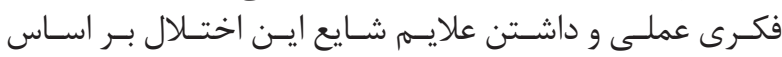

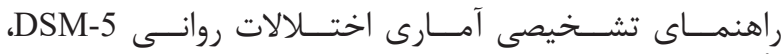

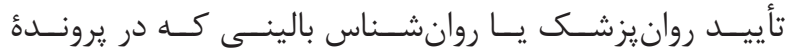

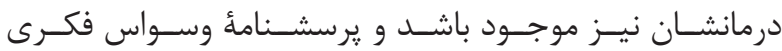

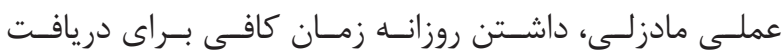

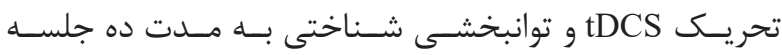

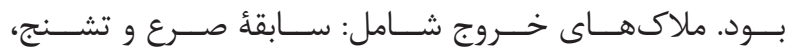

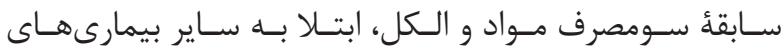

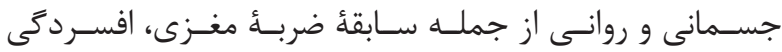

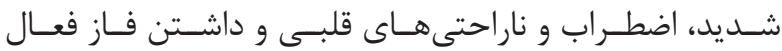

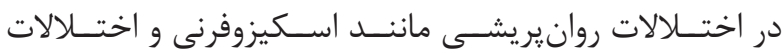

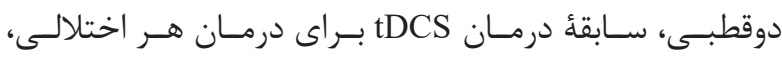

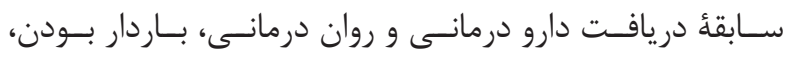

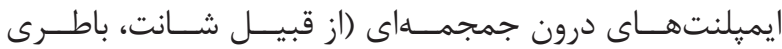

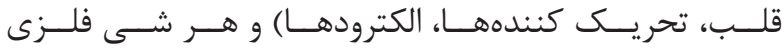

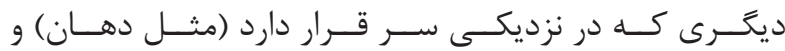

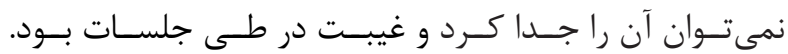

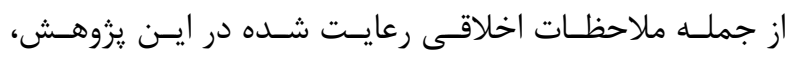

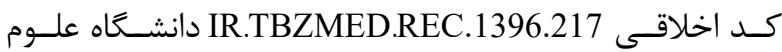

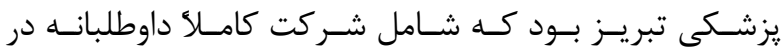

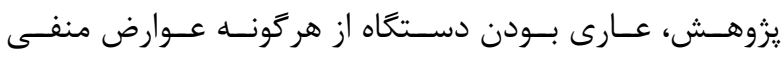

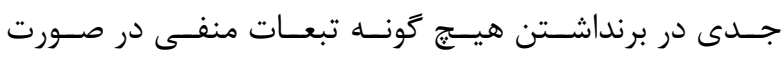

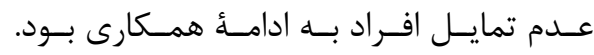

${ }^{20}$ Madzly obsessive- compulsive

${ }^{21}$ Classic stroop test 


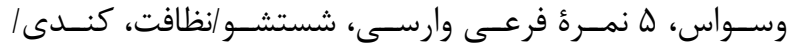

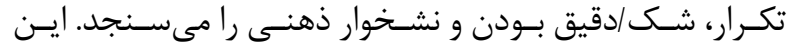

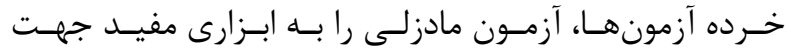

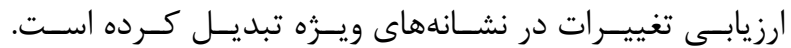

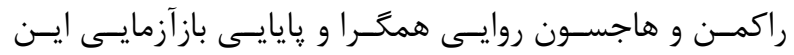

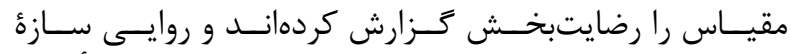

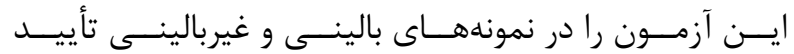

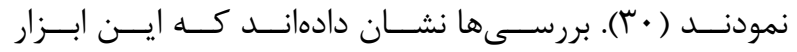

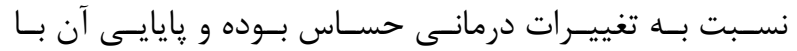

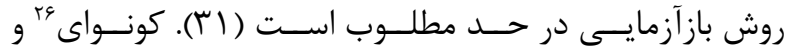

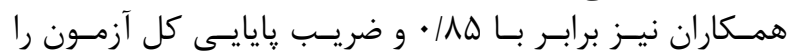

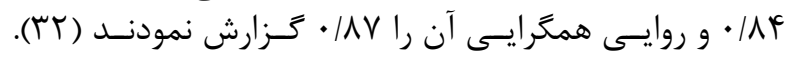

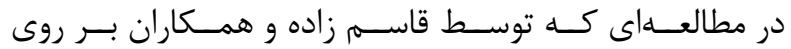

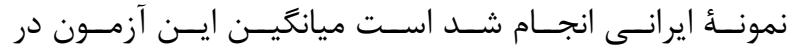

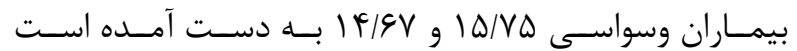

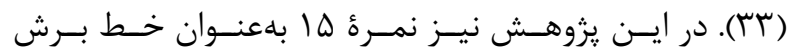

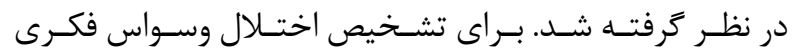

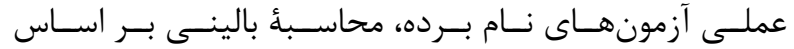

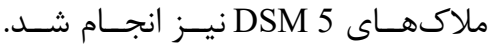

تحريك الكتريكى مستقيم فر اجمجمهاى

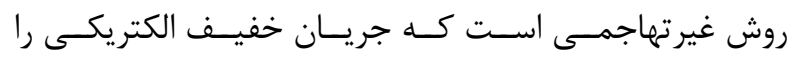

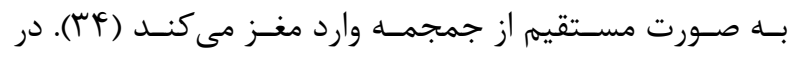

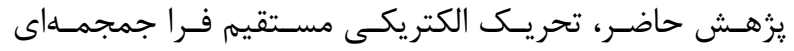

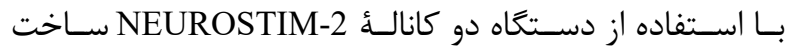

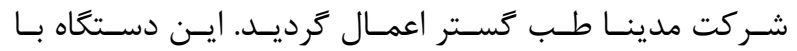

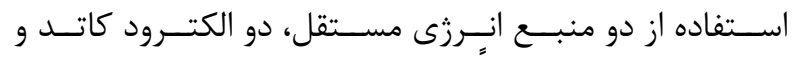

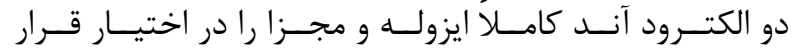

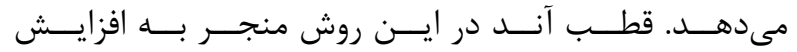

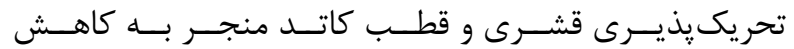

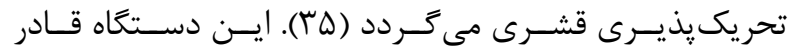

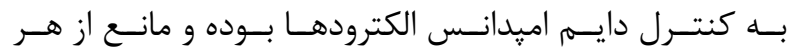

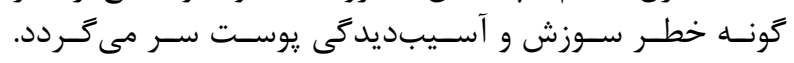

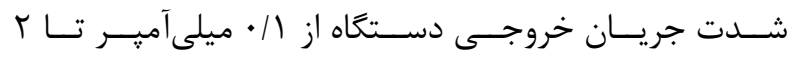

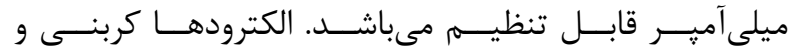

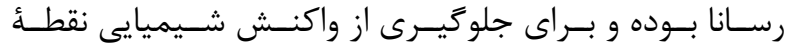

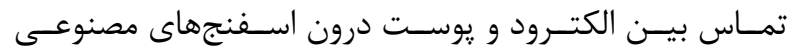

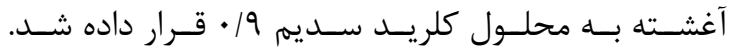

$$
\text { آزمون ماتريس هاى بيشروندهُ ريون }
$$

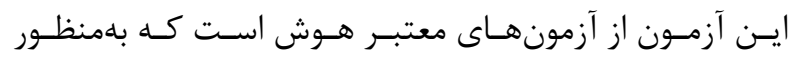

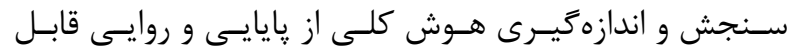

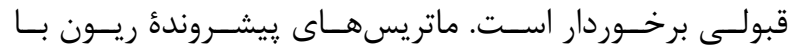

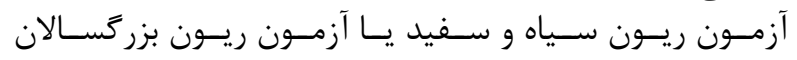

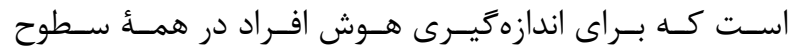

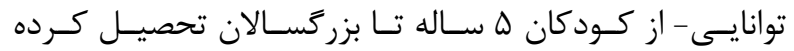

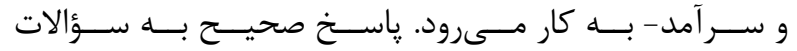

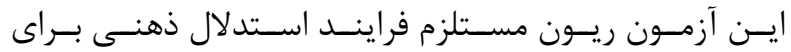

${ }^{22}$ Frontal cortex

${ }^{23}$ Congruent

${ }^{24}$ Incongruent

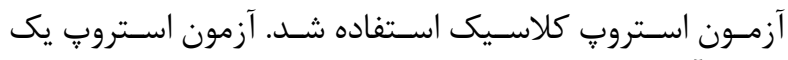

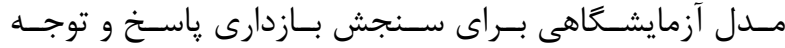

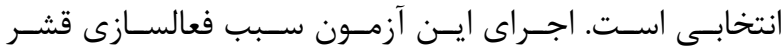

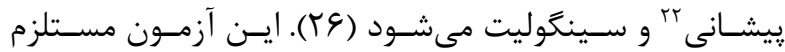

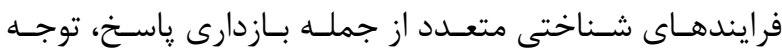

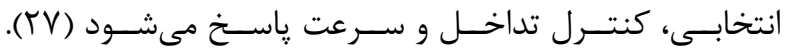

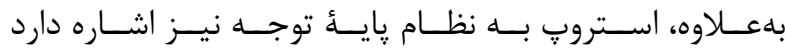

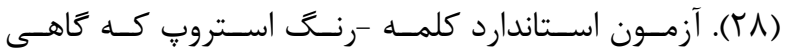

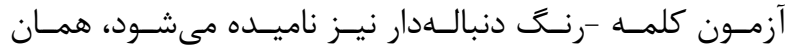

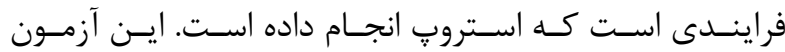

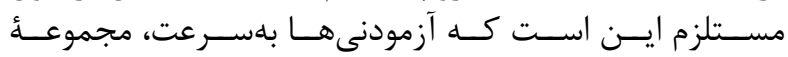

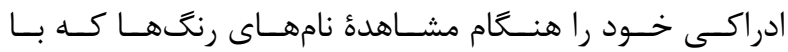

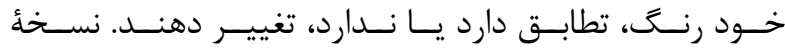

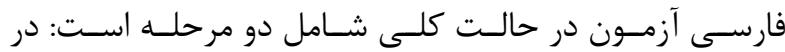

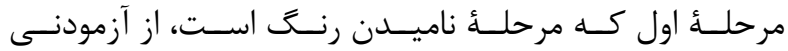

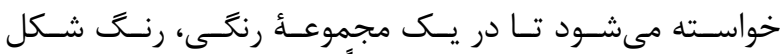

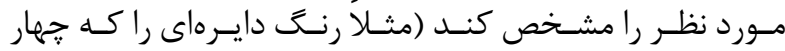

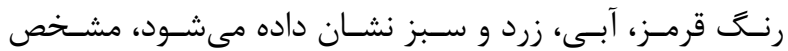

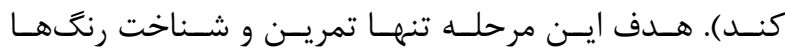

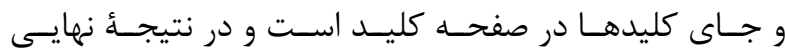

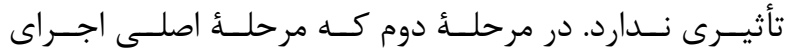

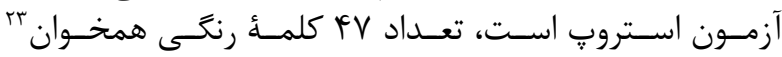

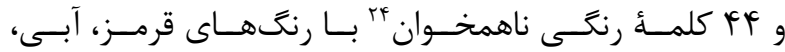

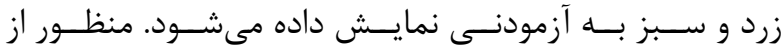

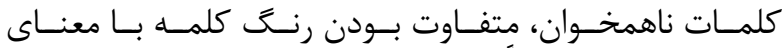

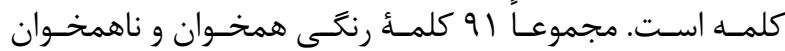

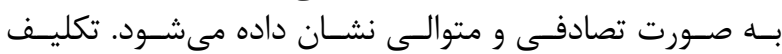

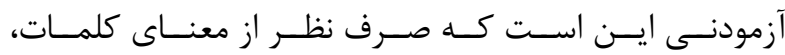

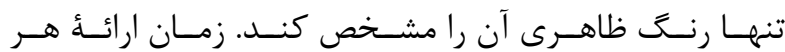

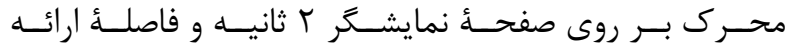

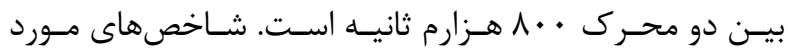

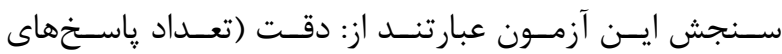

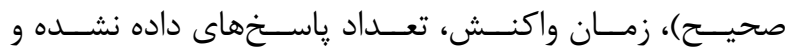

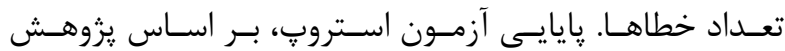

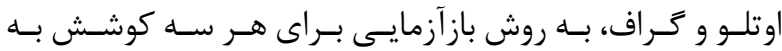

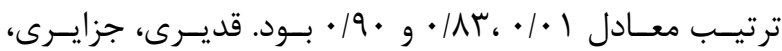

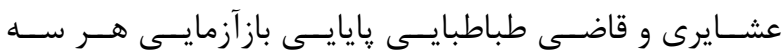

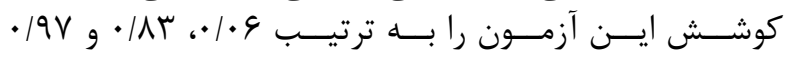

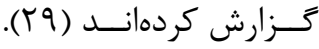

\section{ب - يرسشنامة وسواس فكرى عملى مادزلى}

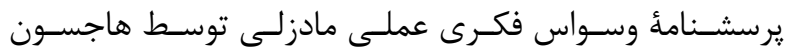

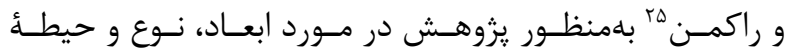

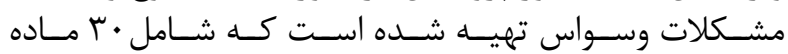

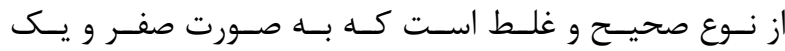

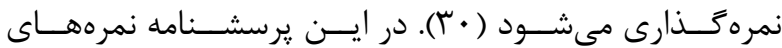

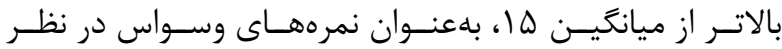

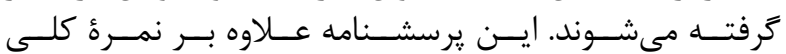

${ }^{25}$ Hodgson and Rachman
${ }^{26}$ Conway 


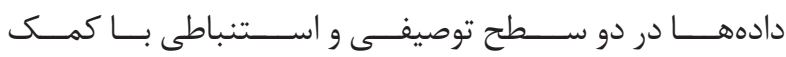

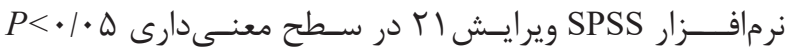

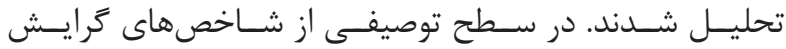

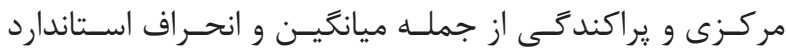

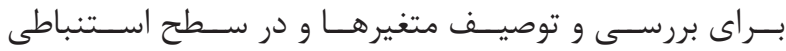

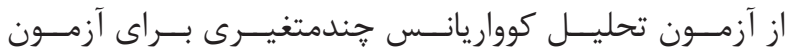

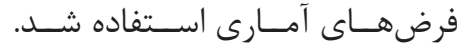

كافته ها

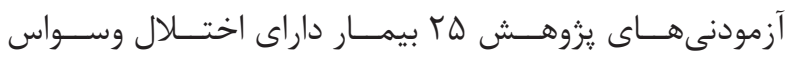

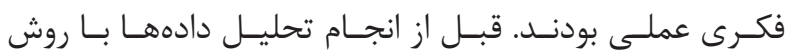

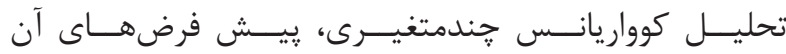

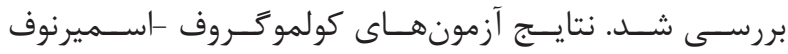

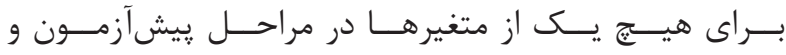

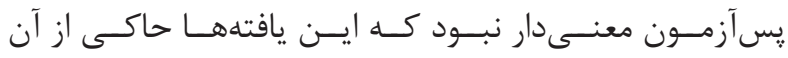

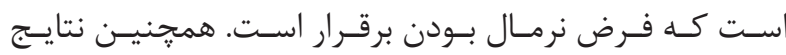

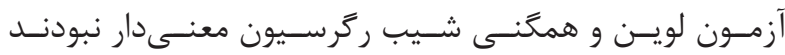

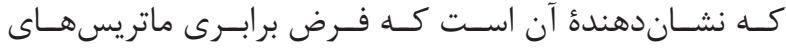

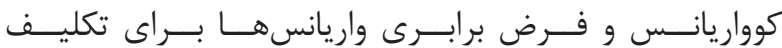

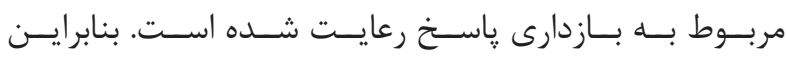

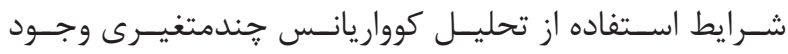

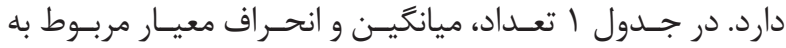

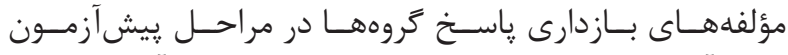

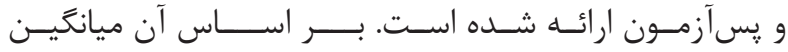

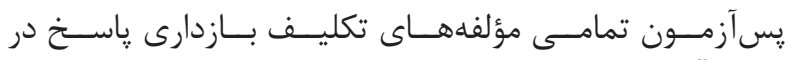

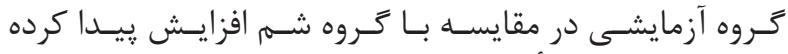

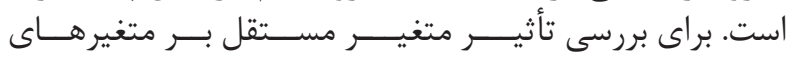

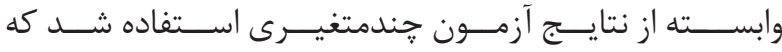

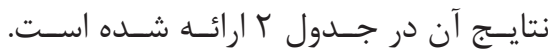

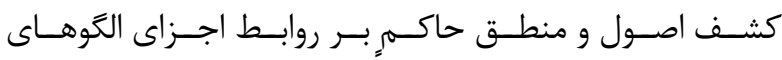

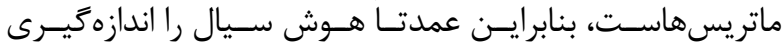

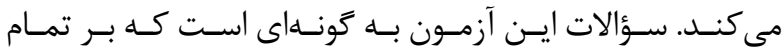

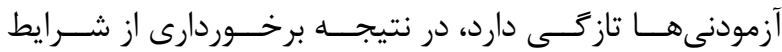

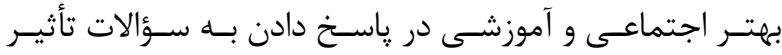

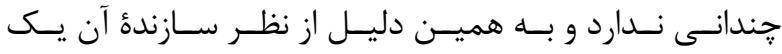

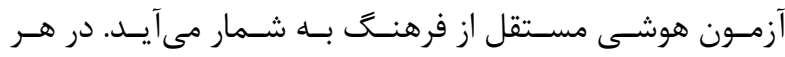

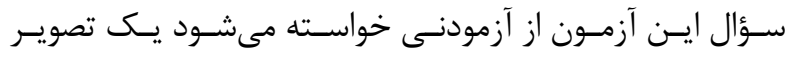

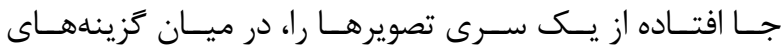

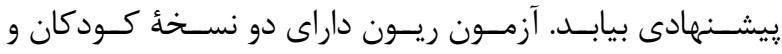

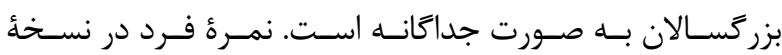

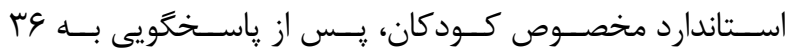

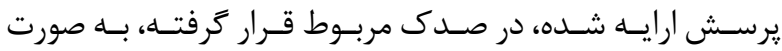

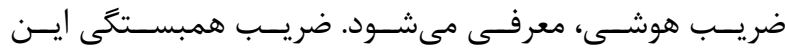

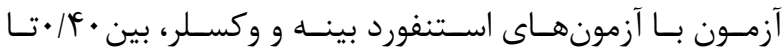

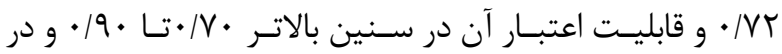

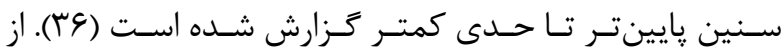

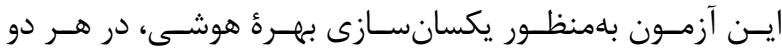

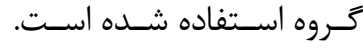

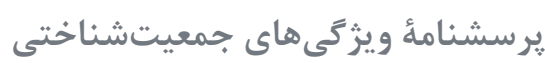

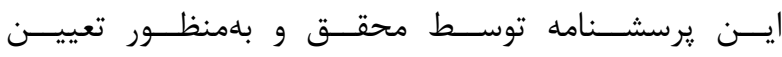

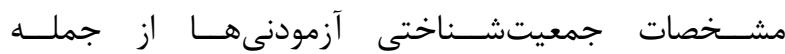

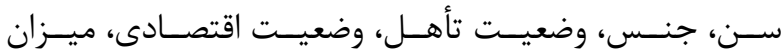

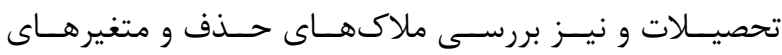

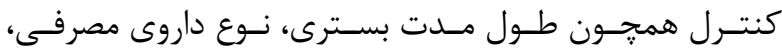

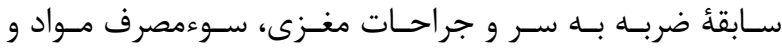

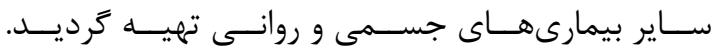
تجزيه و تحليل دادهها

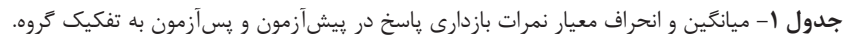

\begin{tabular}{|c|c|c|c|c|c|c|}
\hline \multicolumn{2}{|c|}{ بسآزمون } & \multicolumn{3}{|c|}{ بيش آزمون } & \multicolumn{2}{|c|}{ كروه } \\
\hline انحراف استاندارد & ميانكَين & انحراف استاندارد & ميانكين & تعداد & & \\
\hline$\cdot / A \cdot 1$ & $P 4 / 10$ & $f / r v \mid$ & pripg & ז' & آزمايش & \multirow{2}{*}{ باسخ صحيح همخوان } \\
\hline V/AV & $p q / 1$. & $1 / 1 \% a$ & $\varphi Q / A$. & $\pi$ & شم & \\
\hline $1 / \pi r$ & Fr/Gr & F/AYI & $f \cdot 1 \cdot 1$ & $\pi$ & آزمايش & \multirow{2}{*}{ ياسخ صحيح ناهمخوان } \\
\hline 1/9T & $\mathrm{Fr} / \mathrm{r}$. & $T / f(D)$ & FI/Af & ir & شم & \\
\hline $1 . r / . r$ & VIA/IT & $1 \cdot \Delta / 11$ & V9./9f & זו & آزمايش & \multirow{2}{*}{ زمان باسخ همخوان } \\
\hline$\Delta r / 19$ & VFG/VA & TFITT & $V F * / F$. & ir & شم & \\
\hline $1 . T / F 9$ & A. T/Kr & IrGIAD & $\Lambda 9 \Lambda / r \Lambda$ & ir & آزمايش & \multirow{2}{*}{ زمان باسخ ناهمخوان } \\
\hline I. T/VF & $A F \Delta / 91$ & $11 \pi / 91$ & $\Lambda \in F / A I$ & ir & شم & \\
\hline $1 / 91$ & $-r / \Delta F$ & . $/ 9$ Tा & $-r / v V$ & ir & آزمايش & \multirow{2}{*}{ نمرة تداخل } \\
\hline $1 / \cdot r$ & $-r / r$. & $1 / 1$. & $-\Gamma / 9$. & $\pi$ & شم - ام & \\
\hline
\end{tabular}


جدول r- نتايج آزمون קندمتغيرى براى بررسى اثربخشى درمان تحريك DCS براى بازدارى ياسخ.

\begin{tabular}{|c|c|c|c|c|c|}
\hline اندازة اثر & معنى دارى & F آمارة F & مقدار & آزمونها & متغير مستقل \\
\hline .19 .9 & $.1+41$ & $\Delta / r V r$ & .19 .9 & اثر يِيلايى & \multirow{4}{*}{ روش درمان } \\
\hline .19 .9 & $+1+* 1$ & $\Delta / r V r$ &.$/ 4 q 4$ & لامبداى ويلكز & \\
\hline .19 .9 & $.1 * 1$ & $\Delta / r v r$ & $1 / \Delta r \Delta$ & اثر هتلينت & \\
\hline .19 .9 & $\cdot 1+* 1$ & $\Delta / r V H$ & $1 / \Delta r \Delta$ & بزركترين ريشهروى & \\
\hline
\end{tabular}

در جــــدول ب ارائـــهـ شـــــ

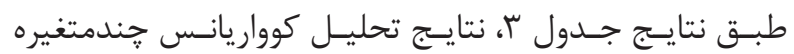

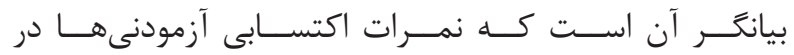

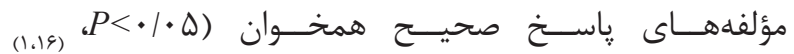

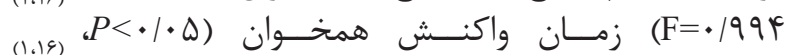

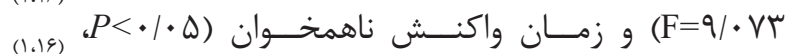

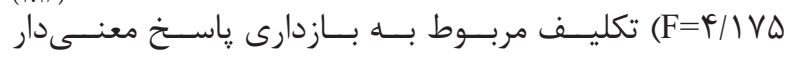

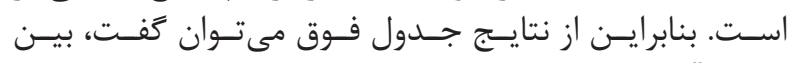

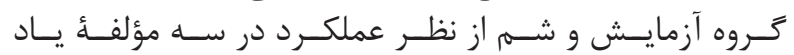

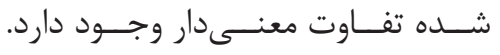

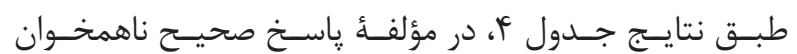

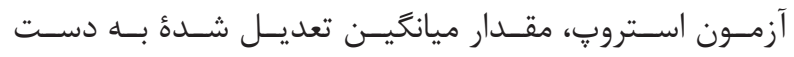

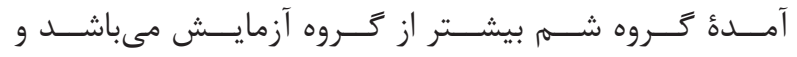

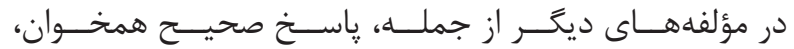

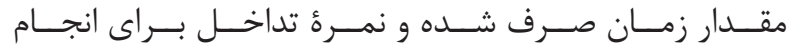

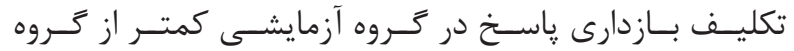

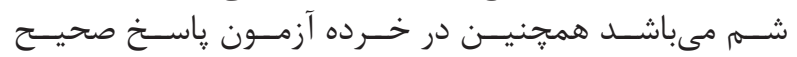

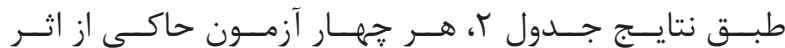

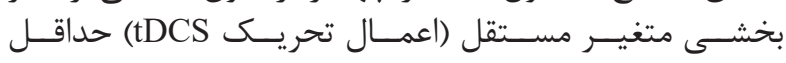

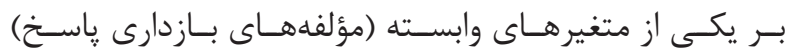

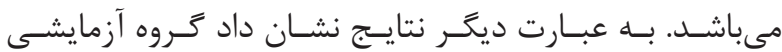

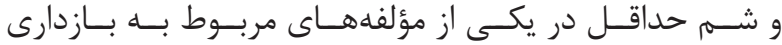

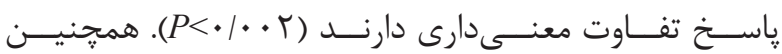

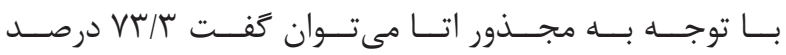

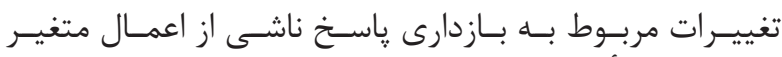

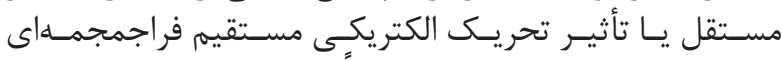

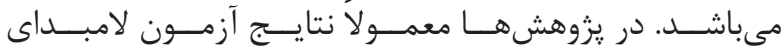

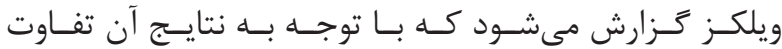

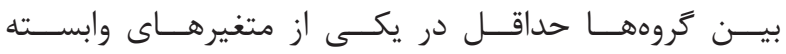

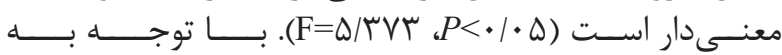

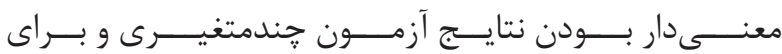

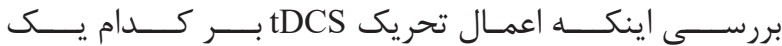

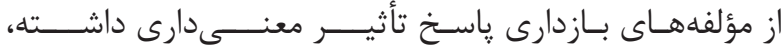

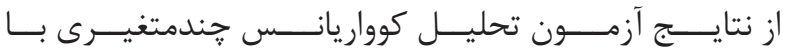

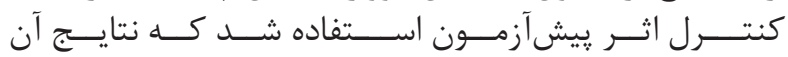

جدول بـ- نتايج تحليل كوواريانس حندمتغيره براى بررسى يروتكل تحريك DCS بر خرده آزمونهاى تكليف بازدارى ياسخ.

\begin{tabular}{|c|c|c|c|c|c|c|c|}
\hline الثاززة ائر & سطح معنى & Fارة F آمارة & مياتئين مجنورات & لرجئة آزادى df & هجموعد مجذور ات & منابع & هتثيرها \\
\hline \multirow[t]{3}{*}{.$/ \pi 11$} & $+1 \cdot T F$ & - /११९ & $\cdot \angle \Delta Y F$ & 1 & $\cdot \Delta \Delta V F$ & كروه & \multirow{3}{*}{ باسخ محيح همخوان } \\
\hline & & & $+\int \Delta V A$ & 19 & $Q / T F D$ & ths & \\
\hline & & & & rr & $F A A$ & كل ل & \\
\hline \multirow[t]{3}{*}{.1 .49} &.$/ V \Delta$ & $\| \mu$ & $+/ 111$ & 1 & $+/ 111$ & كروه & \multirow{3}{*}{ باسخ صحيح ثالمنغوان } \\
\hline & & & $1 / 1 / 9$ & 19 & IA/AY & خطا & \\
\hline & & & & rt & $P+F i$ & كل & \\
\hline \multirow[t]{3}{*}{ •/TST } & 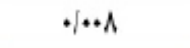 & $9 /+y r$ & $F F+F Y / T A$ & 1 & $F F+F Y / T A$ & 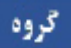 & \multirow{3}{*}{ زعان واكنش همخوان } \\
\hline & & & $F \wedge A F / V G$ & 19 & $V Y G / \pi I$ & خ خطا & \\
\hline & & & & rr & $\mid K A / \Delta A$ & كل ل & \\
\hline \multirow[t]{3}{*}{. /TVY } & $+|+r|$ & $f / / Y \Delta$ & TPYIV/AA & 1 & rFYIY/QA & كزوه & \multirow{3}{*}{ زمان واكتش ناهعخوان } \\
\hline & & & $\Delta 9 r 1 /+99$ & 19 & QFY/DF & خ خ & \\
\hline & & & & rr & $\mid \Delta \Delta / \Delta A$ & 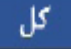 & \\
\hline \multirow[t]{2}{*}{$+1 \cdot 1 \%$} & $+/ \$ F$ &.$|T| F$ & -ITAT & 1 & */TAT & كروه & \multirow{3}{*}{ ثمرة تداخل } \\
\hline & & & $1 / F F$ & 19 & זq/TA & خطا & \\
\hline & & & & rr & $r+9$ & كل & \\
\hline
\end{tabular}




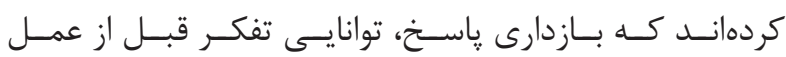

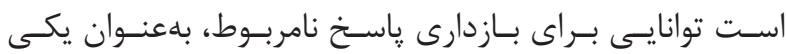

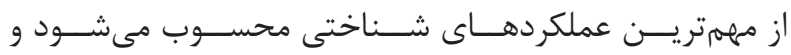

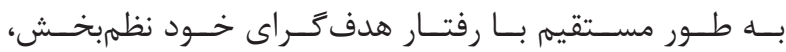

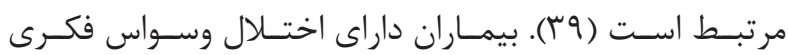

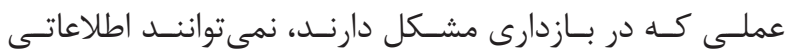

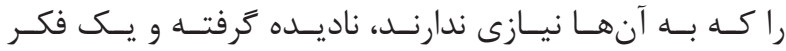

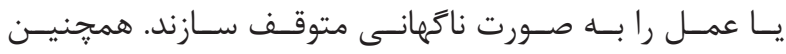

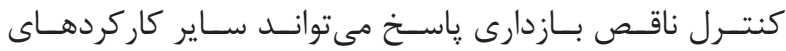

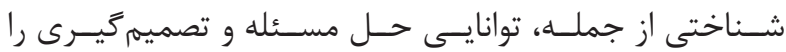

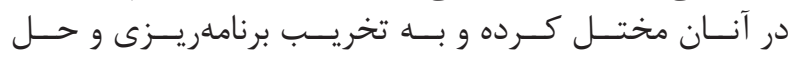

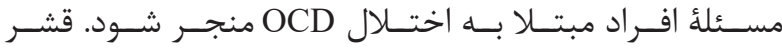

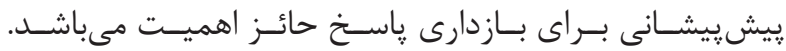

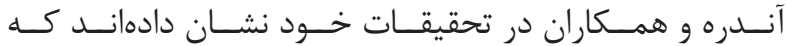

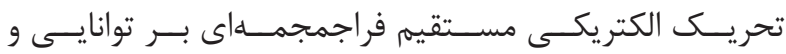

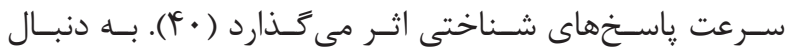

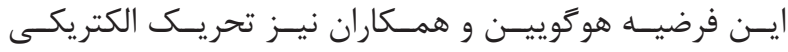

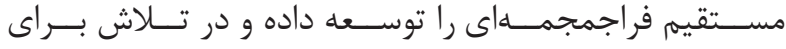

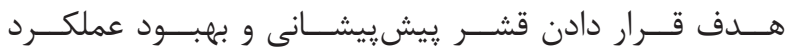

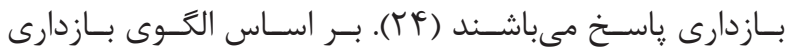

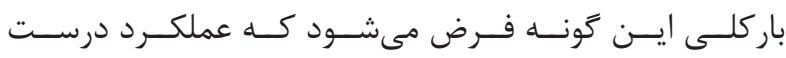

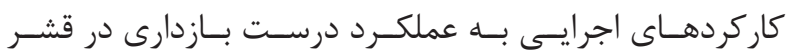

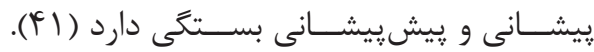

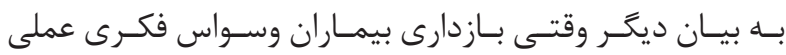

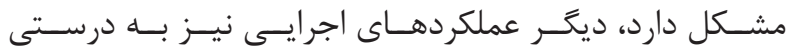

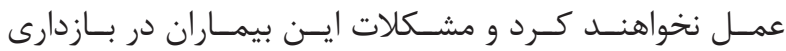

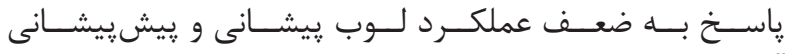

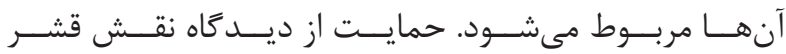

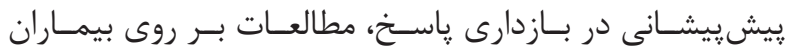

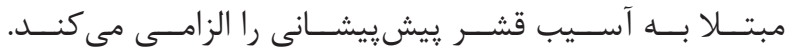

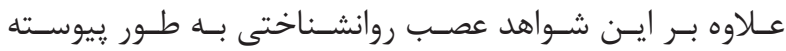

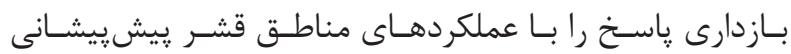

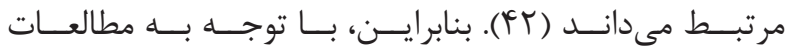

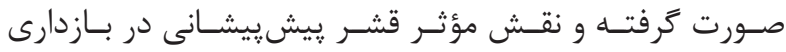

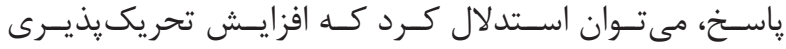

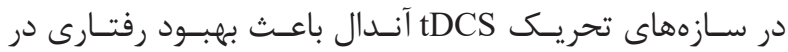

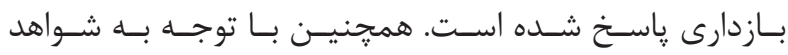

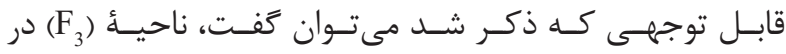

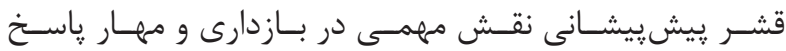

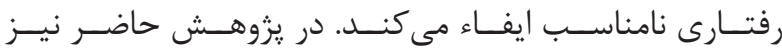

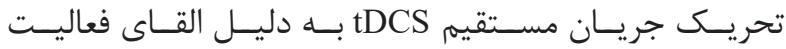

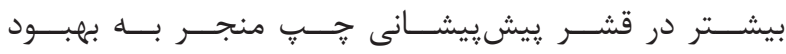

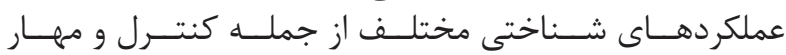

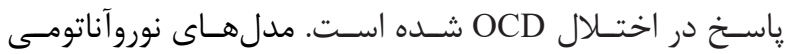

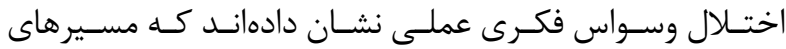

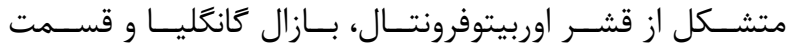

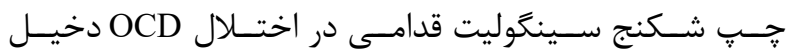

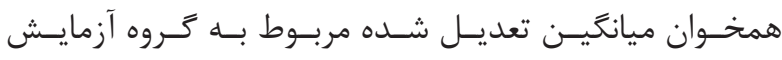

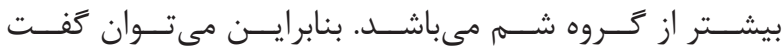

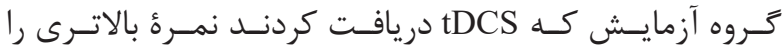

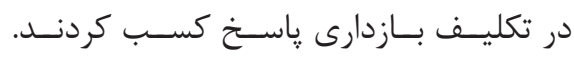
جدول F- ميانگين تعديل شده براى تكليف مربوط به بازدارى پاسخ.

\begin{tabular}{|c|c|c|}
\hline ميانكين & كروه & متغيرها \\
\hline $1 / 994$ & آزمايش & \multirow{2}{*}{ پاسخ صحيح همخوان } \\
\hline $1 / 901$ & شم & \\
\hline I/OTY & آزمايش & \multirow{2}{*}{ باسخ صحيح ناهمخوان } \\
\hline 1/GrA & شم & \\
\hline$r / Y \in \Lambda$ & آزمايش & \multirow{2}{*}{ مياتكين زمان واكنش همخوان } \\
\hline$r / 9 \ldots$ & شم & \\
\hline$r / 9 \cdot f$ & آزمايش & \multirow{2}{*}{ ميانكَين زمان واكنش ناهمخوان } \\
\hline r/QT. & شم & \\
\hline$-T / \lambda \cdot V$ & آزمايش & \multirow{2}{*}{ نمرة تداخل } \\
\hline$-f(\lambda \Delta)$ & شم & \\
\hline
\end{tabular}

بحث و نتيجهَ كيرى

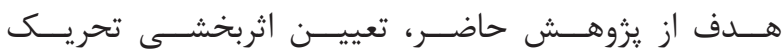

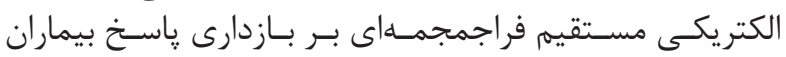

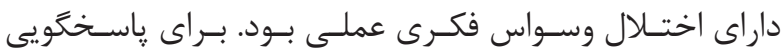

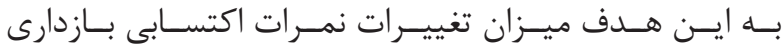

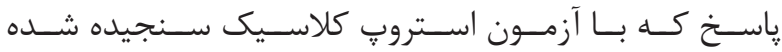

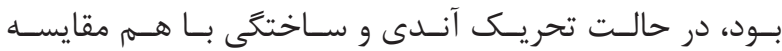

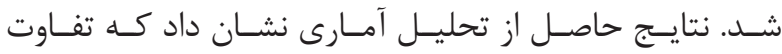

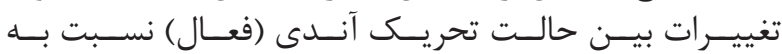

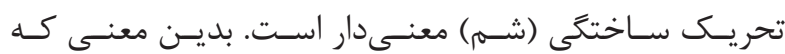

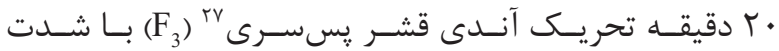

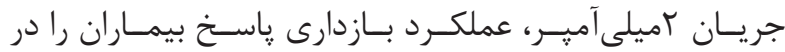

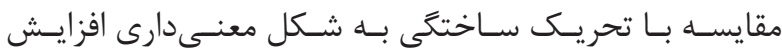

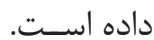

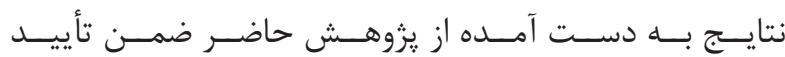

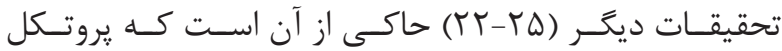

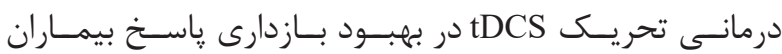

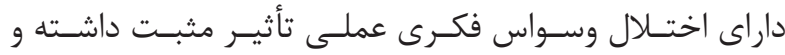

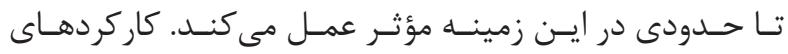

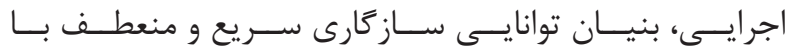

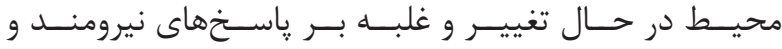

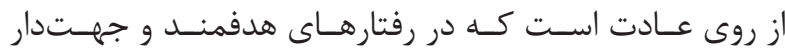

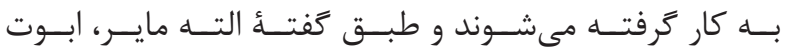

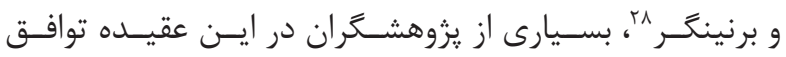

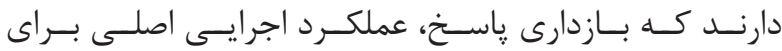

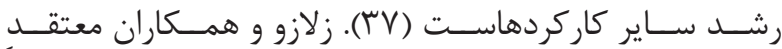

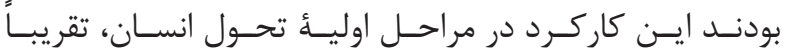

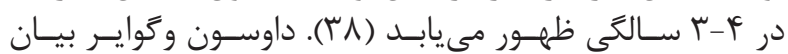




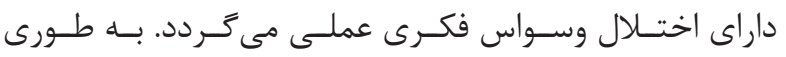

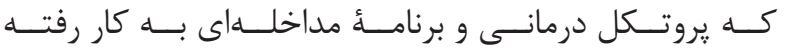

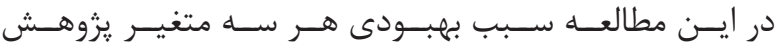

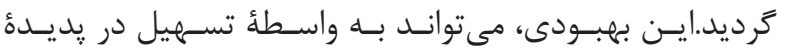

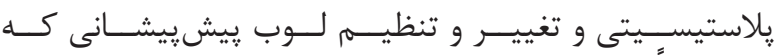

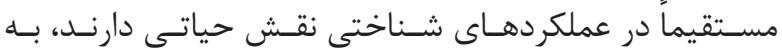

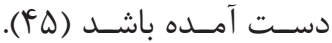

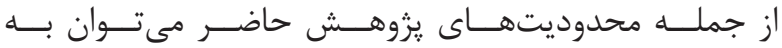

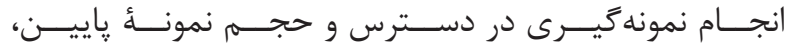

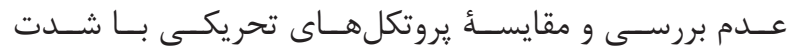

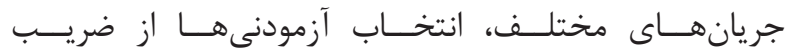

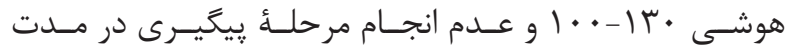

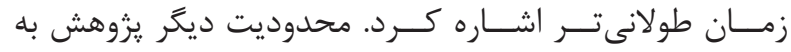

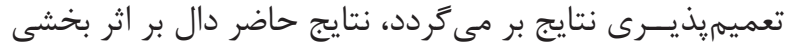

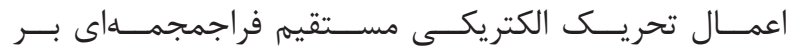

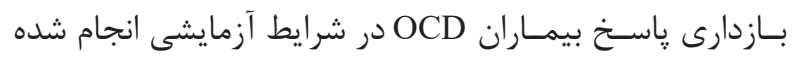

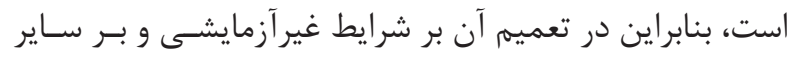

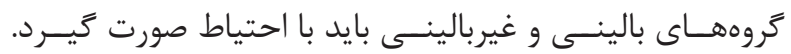

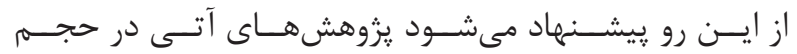

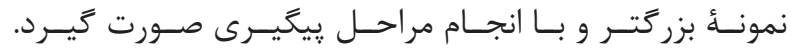

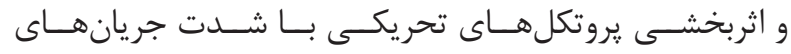

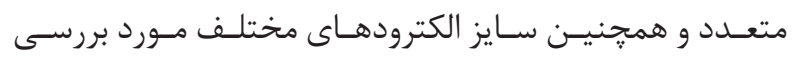

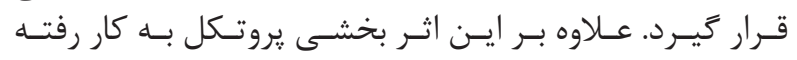

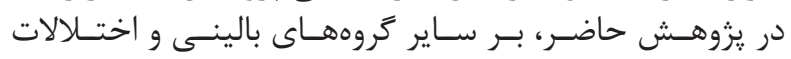

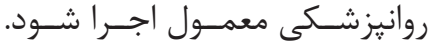

$$
\begin{aligned}
& \text { تشكر و قدردانى }
\end{aligned}
$$

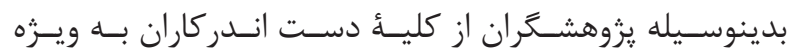

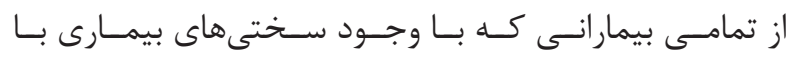

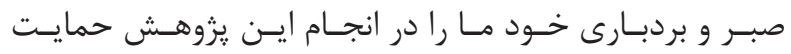

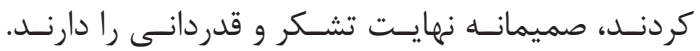

1. American Psychiatric Association (APA). The diagnostic and statistical manual of mental disorders(DSM-5). $5^{\text {th }}$ ed. Wasington, DC: American Psychiat Association, Published. 2013.

2. Real E, Montejo A, Alonso P, Menchon JM. Sexuality and obsessive-compulsive disorder: the hidden affair. Neuropsychiat Res. 2013; 3(1): 23-31.

3. Grados MA, Walkup J, Walford S. Genetics of obsessive-compulsive disorders: new findings and challenges. Brain Dev. 2003; 25: S55-S61.

4. Kühn S, Kaufmann C, Simon D, Endrass T, Gallinat J, Kathmann N. Reduced thickness of anterior cingulate cortex in obsessive-compulsive disorder. Cortex. 2013; 49(8): 2178-85.

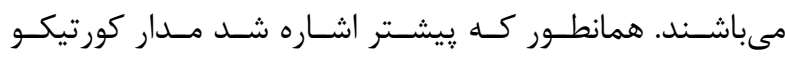

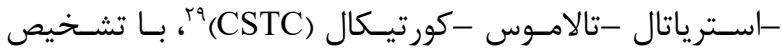

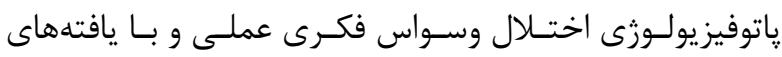

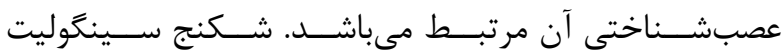

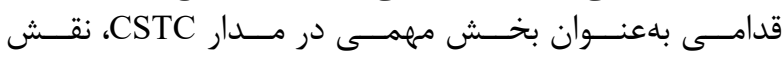

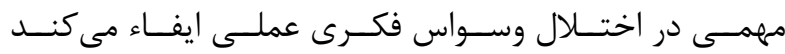

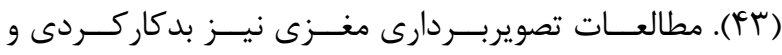

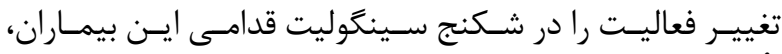

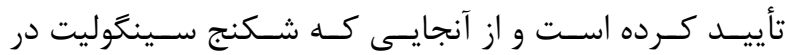

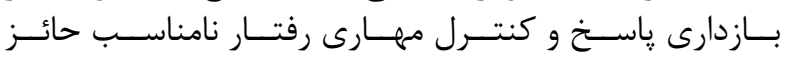

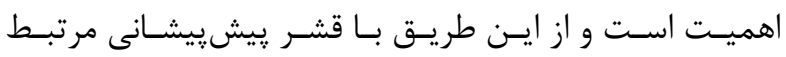

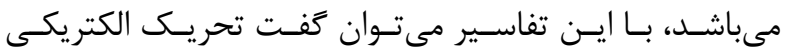

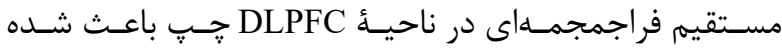

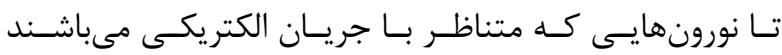

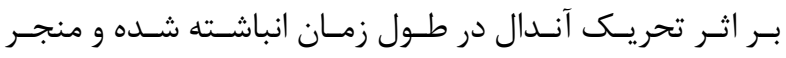

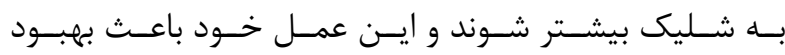

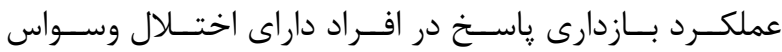

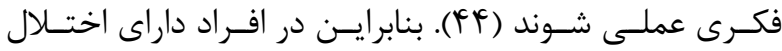

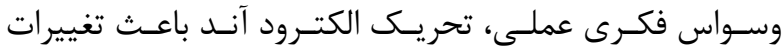

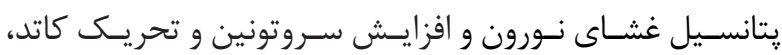

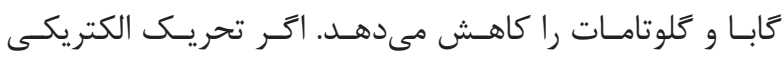

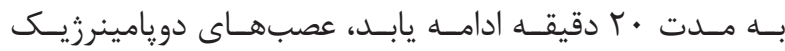

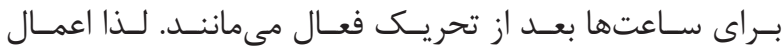

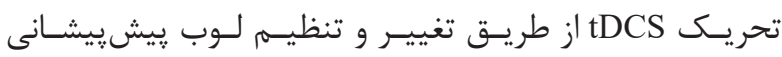

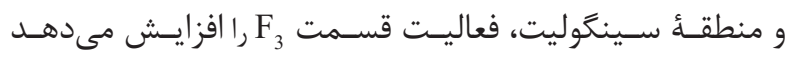

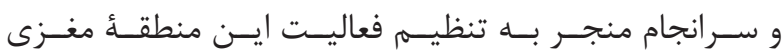

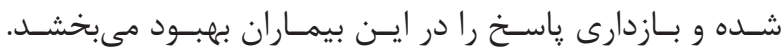

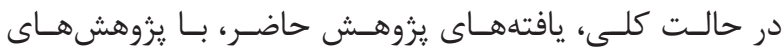

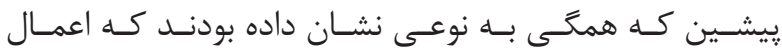

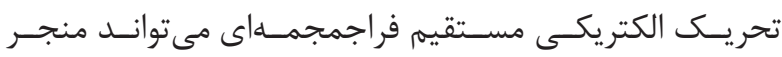

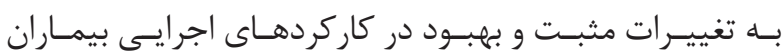

منابع

5. Grant JE, Pinto A, Gunnip M, Mancebo MC, Eisen JL, Rasmussen SA. Sexual obsessions and clinical correlates in adults with obsessive-compulsive disorder. Compr Psychiatry. 2006; (5)47: 325-9.

6. Kuelz AK, Hohagen F, Voderholzer U. Neuropsychological performance in obsessive compulsive disorder: a crtical review. Bio Psychol. 2004; 65(3): 185-236.

7. Aourizerate B, Guehl D, Cuny E, Rougier A, Biolac B Tignol J. Pathophysiology of obsessive- compulsive disorder: A necessary link between phenomenology, neuropsychology, Imagery and physiology. Prog Neurobiol. 2004; 72(3): 195-221.

8. Gruner P, Pittenger C. Cognitive inflexibility in

${ }^{29}$ Cortico striatal thalamic cortical circut 
obsessive- compulsive disorder. Neuroscience. 2017; 345: 243-55.

9. Evans D, Leckman JF. Origins of obsessivecompulsive disorders: developmental and evolutionary perspective. J Dew psycho. 2006; 72: 321-332.

10. Menzies L, Achard S, Chamberlain SR, Fineberg $\mathrm{N}$, Chen $\mathrm{CH}$, del Campo $\mathrm{N}$, et al. Neurocognitive endophenotypes of obsessive-compulsive disorder. Brain. 2007; 130: 3223-36.

11. Chamberlain SR, Blackwell AD, Fineberg NA, Robbins TW, Sahakian BJ. The neuropsychology of obsessive compulsive disorder: the importance of failures in cognitive and behavioural inhibition as candidate endophenotypic markers. Neurosci \& Biobehav Rev. 2005; 29(3): 399-419.

12. Rao NP, Reddy YJ, Kumar KJ, Kandavel T, Chandrashekar C. Are neuropsychological deficits trait markers in OCD? Prog Neuropsychopharmacol Biol Psychiatry. 2008; 32(6): 1574-9.

13. Shin N, Lee T, Kim E, Kwon J. Cognitive functioning in obsessive-compulsive disorder: a meta-analysis. Psychol med Sci. 2014; 44(6): 1121-30.

14. Purcell R, Maruff P, Kyrios M, Pantelis C. Cognitive deficits in obsessive-compulsive disorder on tests of frontal-striatal function. Biol Psychiatry. 1998; 43(5): 348-57.

15. Brewin C, Beaton A. Thought suppression, intelligence, and working memory capacity. Behav Res thera. 2002; 40(8): 923-30.

16. Demeter G, Csigo K, Harsanyi A, Nemeth A, Racsmany M. Impaired executive function in obsessive compulsive disorder (OCD). Psychiatr Hung. 2008; 23(2): 85-93.

17. Degeus F, Denys DA, Sitskoormm Westenberg HG. Attention and cognition in patients with obsessive compulsive disorder. Psychiatry Clin Neurosci. 2007; 67(1): 45-53.

18. Park HS, Shin YW, Ha TH, Shin MS, Kim YY, Lee $\mathrm{YH}$, et al. Effect of cognitive training focusing on organizational strategies in patients with obsessivecompulsive disorder. Psychiatry Clin Neurosci. 2006; 60(6): 718-26.

19. Buhlmann U, Decker Sbach T, Engelhard I, Cook LM, Rauch SL, Kathmann N, et al. Cognitive retraining for organizational impairment in obsessive- compulsive disorder. Psychiatry Res. 2006; 144(2-3): 109-16.
20. Bannon S, Gonsalvez CJ, Croft RJ, Biyce PM. Executive functions in ibsessive- compulsive disorder: state or trait deficits? Aust N Z J Psychiatry. 2006; 40(11-12): 1031-8.

21. Clark VP, Coffman BA, Trumbo MC, Gasparovic C. Transcranial direct current stimulation (tDCS) produces localized and specific alterations in neurochemistry: a $1 \mathrm{H}$ magnetic resonance spectroscopy study. Neurosci Lett. 2011; 500(1): 67-71.

22. Alizadeh Goradel J, Pouresmali A, Mowlaie M, Sadeghi Movahed F. The effects of transcranial direct current stimulation on obsession-compulsion, anxiety, and depression of a patient suffering from Obsessivecompulsive Disorder. Practice in Clinical Psychology. 2016; 4(2): 75-80.

23. Dinn WM, Aycicegi-Dinn A, Göral F, Karamursel S, Yildirim EA, Hacioglu-Yildirim M, et al. Treatmentresistant obsessive-compulsive disorder: Insights from an open trial of transcranial direct current stimulation (tDCS) to design a RCT. Neurol, Psychiat and Brain Res. 2016; 22(3-4): 146-54.

24. Hogeveen J, Grafman J, Aboseria M, David A, Bikson M, Hauner K. Effects of high-definition and conventional tDCS on response inhibition. Brain Stimul. 2016; 9(5): 720-9.

25. Narayanaswamy JC, Jose D, Chhabra H, Agarwal SM, Shrinivasa B, Hegde A, et al. Successful application of add-on transcranial direct current stimul (tDCS) for treatment of SSRI resistant OCD. Brain Stimul: 2015; 8(3): 655-7.

26. Swick D, Jovanovic J. Anterior cingulate cortex and the Stroop task: neuropsychological evidence for topographic specificity. Neuropsychologia. 2002; 40(8): 1240-53.

27. Solís-Ortiz S, Pérez-Luque E, Morado-Crespo L, Gutiérrez-Muñoz M. Executive functions and selective attention are favored in middle-aged healthy women carriers of the $\mathrm{Val} / \mathrm{Val}$ genotype of the catechol-omethyltransferase gene: a behavioral genetic study. Behav Brain Funct. 2010; 6(1): 67. doi: 10.1186/17449081-6-67.

28. Groth-Marnat GE. Neuropsychological assessment in clinical practice: a guide to test interpretation and integration. John Wiley \& Sons Inc. 2000; p. 672.

29. Ghadiri F, Jazayeri A, Aashayeri H, Ghazi Tabatabai M. Deficit in Executive Functioning in Patients With Schizo-Obsessive Disorder. Journal of Advances in Cognitive Sciences. 2006; 8(3): 11-24. 
30. Hodgson RJ, Rachman S. Obsessional-compulsive complaints. Behav Res Ther. 1977; 15(5): 389-95.

31. Emmelkamp P, Beens H. Cognitive therapy with obsessive- compulsive disorder: a comparative evaluation. Behav Res Ther. 1991; 29(3): 293-300.

32. Conway M, Csank PA, Holm SL, Blake CK. On assessing individual differences in rumination on sadness. J Pers Assess. 2000; 75(3): 404-25.

33. Ghasemzadeh H. Cognitive- behavioral model of OCD with emphasis on encountering and emotional processing. 118th seminar on neuro-cognitive psychology. Shohada Medical center. Shahid Beheshti University of Medical Sciences. 2003; 8(2): 25-40.

34. Nitsche MA, Cohen LG, Wassermann EM, Priori A, Lang N, Antal A, et al. Transcranial direct current stimulation: state of the art 2008. Brain Stimul. 2008; 1(3): 206-23.

35. Fregni F, Boggio PS, Nitsche M, Bermpohl F, Antal A, Feredoes E, et al. Anodal transcranial direct current stimulation of prefrontal cortex enhances working memory. Exp Brain Res. 2005; 166(1): 23-30.

36. Sharifi T, Rabee M. The use of 4th edition of Wechsler exam for kids in recognizing disorders in writing and mathmatics. Journal of Research in Learning Disabilities. 2012; 2(2): 59-75.

37. Altemeier LE, Abbott RD, Berninger VW. Executive functions for reading and writing in typical literacy development and dyslexia. J Clin Exp Neuropsychol. 2008; 30(5): 588-606.

38. Zelazo PD. The dimensional change card sort (dccs): a method of assessing executive function in children. Nat Protoc. 2006; 1(1): 297-301.

39. Dawson P, Guare R. Executive skills in children and adolescents: a practical guide to assessment and intervention: New York: Guilford Press .2010.

40. André S, Heinrich S, Kayser F, Menzler K, Kesselring J, Khader PH, et al. At-home tDCS of the left dorsolateral prefrontal cortex improves visual shortterm memory in mild vascular dementia. J Neurol Sci. 2016; 369: 185-90.

41. Barkley RA. Behavioral inhibition, sustained attention, and executive functions: constructing a unifying theory of ADHD. Psychol Bull. 1997; 121(1): 65-94.

42. Flöel A, Suttorp W, Kohl O, Kürten J, Lohmann H, Breitenstein $\mathrm{C}$, et al. Non-invasive brain stimulation improves object-location learning in the elderly. Neurobiol Aging. 2012; 33(8): 1682-9.

43. Yun GJ, Chun MH, Kim BR. The effects of transcranial direct-current stimulation on cognition in stroke patients. J Stroke. 2015; 17(3): 354-8.

44. Rosenberg DR, Mirza Y, Russell A, Tang J, Smith $\mathrm{JM}$, Banerjee SP, et al. Reduced anterior cingulate glutamatergic concentrations in childhood OCD and major depression versus healthy controls. J Am Acad Child Adolesc Psychiatry. 2004; 43(9): 1146-53.

45. Bolton J, Moore GJ, MacMillan S, Stewart CM, Rosenberg DR. Case study: caudate glutamatergic changes with paroxetine persist after medication discontinuation in pediatric OCD. J Am Acad Child Adolesc Psychiatry. 2001; 40(8): 903-6. 